\title{
Transplantation of induced neural stem cells (iNSCs) into chronically demyelinated corpus callosum ameliorates motor deficits
}

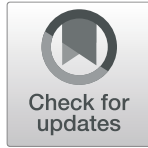

\author{
Genevieve M. Sullivan ${ }^{1}$, Andrew K. Knutsen ${ }^{2,3}$, Luca Peruzzotti-Jametti ${ }^{4}$, Alexandru Korotcov ${ }^{2,3}$, \\ Asamoah Bosomtwi ${ }^{2,3}$, Bernard J. Dardzinski ${ }^{2,3}$, Joshua D. Bernstock ${ }^{4,5}$, Sandra Rizzi ${ }^{6}$, Frank Edenhofer ${ }^{6+}$, \\ Stefano Pluchino ${ }^{4 \dagger}$ and Regina C. Armstrong ${ }^{1,2^{*}}$ (D)
}

\begin{abstract}
Multiple Sclerosis (MS) causes neurologic disability due to inflammation, demyelination, and neurodegeneration. Immunosuppressive treatments can modify the disease course but do not effectively promote remyelination or prevent long term neurodegeneration. As a novel approach to mitigate chronic stage pathology, we tested transplantation of mouse induced neural stem cells (iNSCs) into the chronically demyelinated corpus callosum (CC) in adult mice. Male C57BL/6 mice fed 0.3\% cuprizone for 12 weeks exhibited CC atrophy with chronic demyelination, astrogliosis, and microglial activation. Syngeneic iNSCs were transplanted into the CC after ending cuprizone and perfused for neuropathology 2 weeks later. Magnetic resonance imaging (MRI) sequences for magnetization transfer ratio (MTR), diffusion-weighted imaging (T2), and diffusion tensor imaging (DTI) quantified CC pathology in live mice before and after iNSC transplantation. Each MRI technique detected progressive CC pathology. Mice that received iNSCs had normalized DTI radial diffusivity, and reduced astrogliosis post-imaging. A motor skill task that engages the CC is Miss-step wheel running, which demonstrated functional deficits from cuprizone demyelination. Transplantation of iNSCs resulted in marked recovery of running velocity. Neuropathology after wheel running showed that iNSC grafts significantly increased host oligodendrocytes and proliferating oligodendrocyte progenitors, while modulating axon damage. Transplanted iNSCs differentiated along astrocyte and oligodendrocyte lineages, without myelinating, and many remained neural stem cells. Our findings demonstrate the applicability of neuroimaging and functional assessments for pre-clinical interventional trials during chronic demyelination and detect improved function from iNSC transplantation. Directly reprogramming fibroblasts into iNSCs facilitates the future translation towards exogenous autologous cell therapies.
\end{abstract}

Keywords: Neural stem cells, Remyelination, Cuprizone, Multiple sclerosis, Stem cell therapeutics, Diffusion tensor imaging, Magnetization transfer ratio, Magnetic resonance imaging, Reprogramming

\footnotetext{
*Correspondence: regina.armstrong@usuhs.edu

${ }^{\dagger}$ Frank Edenhofer and Stefano Pluchino contributed equally to this work.

'Department of Anatomy, Physiology and Genetics, F. Edward Hebert School

of Medicine, Uniformed Services University of the Health Sciences, 4301

Jones Bridge Rd, Bethesda, MD 20814, USA

${ }^{2}$ Center for Neuroscience and Regenerative Medicine, F. Edward Hebert

School of Medicine, Uniformed Services University of the Health Sciences, Bethesda, MD 20814, USA

Full list of author information is available at the end of the article
}

(C) The Author(s). 2020 Open Access This article is licensed under a Creative Commons Attribution 4.0 International License, which permits use, sharing, adaptation, distribution and reproduction in any medium or format, as long as you give appropriate credit to the original author(s) and the source, provide a link to the Creative Commons licence, and indicate if changes were made. The images or other third party material in this article are included in the article's Creative Commons licence, unless indicated otherwise in a credit line to the material. If material is not included in the article's Creative Commons licence and your intended use is not permitted by statutory regulation or exceeds the permitted use, you will need to obtain permission directly from the copyright holder. To view a copy of this licence, visit http://creativecommons.org/licenses/by/4.0/ The Creative Commons Public Domain Dedication waiver (http://creativecommons.org/publicdomain/zero/1.0/) applies to the data made available in this article, unless otherwise stated in a credit line to the data. 


\section{Introduction}

Recent epidemiological data identified nearly a million adults in the United States alone who are living with a diagnosis of multiple sclerosis (MS), which is almost double prior estimates [1]. This increased appreciation of the MS burden to patients and society emphasizes the urgent need for developing effective treatments. Early in their disease course, relapsing-remitting MS patients often experience periods of autoimmune remission accompanied by spontaneous remyelination [2]. In contrast, the progressive pathology of chronic MS results in sclerotic lesions with axon loss and failed remyelination [3]. Novel treatment strategies are critical to suppress immune attacks, overcome factors limiting remyelination, and prevent neurodegeneration [4-6]. The current studies test a novel neural stem cell-based therapy in an experimental model of chronic demyelination.

Immature oligodendrocyte lineage cells persist in adult human white matter but fail to mature and remyelinate in chronic MS lesions [7-9]. Among interventions to promote remyelination, anti-Lingo-1 and clemastine have advanced to clinical trials in relapsing MS patients $[10,11]$. Anti-Lingo-1 and clemastine act by modulating molecular signals that regulate oligodendrocyte differentiation [1214]. Pre-clinical studies, including those for anti-Lingo-1 and clemastine, have often used acute demyelination models that undergo efficient spontaneous remyelination by endogenous oligodendrocyte progenitor cells (OPCs) $[12,14,15]$. The outcome of these acute stage studies is then based on accelerating remyelination to enhance recovery of function and protect demyelinated axons from further damage. However, acute demyelination models cannot effectively evaluate treatment strategies for chronic demyelination and neurodegeneration [16].

The cuprizone (CPZ) model of corpus callosum (CC) demyelination is well characterized for remyelination studies $[17,18]$. CPZ ingestion for $4-6$ weeks produces acute demyelination with robust OPC amplification and extensive remyelination $[19,20]$. In CPZ and other acute demyelination models, multiple signaling molecules promote remyelination from newly generated oligodendrocytes [2, 21]. Prolonging CPZ ingestion for 10-12 weeks produces chronic demyelination with progressive pathology, including CC atrophy, persistent astrogliosis, OP depletion, and limited remyelination [20, 22-24]. Importantly, CC atrophy correlates with functional outcomes in MS patients [25, 26]. Relevant to modeling sclerotic lesions, chronic CPZ lesions express molecules associated with astrogliosis that inhibit OP differentiation and limit remyelination [23, 27, 28].

The objective of the current study was to employ a combination of translational approaches to evaluate induced neural stem cell (iNSC) transplantation after chronic demyelination. Direct conversion of mouse or human somatic cells to generate iNSCs circumvents the pluripotent stage associated with increased tumorigenesis and genomic instability, with potential as an autologous therapy [29-32]. To model an autologous set-up, we transplanted syngeneic iNSCs into the CC after 12 weeks of $\mathrm{CPZ}$ in two cohorts of mice. In one cohort, longitudinal magnetic resonance imaging (MRI) demonstrated the progression of CC pathology throughout the course of CPZ demyelination, and during recovery after iNSC transplantation. In the second cohort, functional testing used Missstep wheel running to engage the $C C$ and reveal motor deficits after CPZ demyelination [33-35]. Following MRI and behavioral assessments, brain tissue sections were examined for CC atrophy, remyelination, and cellular responses of both transplanted iNSCs and host endogenous cells. Our work provides evidence ofimproved function after iNSC transplantation together with technical advances for translational chronic CPZ model and may pave the way for a novel therapeutic strategy to treat progressive MS by autologous neural stem cell therapies.

\section{Materials and methods \\ Experimental design and statistical analysis}

The experiments were designed as pre-clinical trials to test the effect of iNSC transplantation during chronic $\mathrm{CPZ}$ demyelination. Experiments were carried out according to the Animal Research Reporting: In Vivo Experiments (ARRIVE) guidelines. Technique validation was performed using pilot cohorts. Two parallel trial experiments each used a non-invasive assessment, MRI (structural) or behavior (functional), as the primary outcome measure. A pre-determined study design was used that stated inclusion/exclusion criteria, randomization procedures, and statistical analyses. All experiments were performed with blinding during implementation and analysis. Sample size estimates were based on prior experiments using each primary outcome measure (MRI or behavior). To reduce variability due to the demyelination produced by $\mathrm{CPZ}$, mice were split into groups balanced by weights and assigned to treatment condition using the Excel RAND command for randomization. After completion of the primary outcome assessment, mice in each experiment were perfused for neuropathology and cell type analyses.

Timelines for the mouse cohorts are shown in the corresponding figures and mouse sample numbers are provided in each legend. A total of 100 mice were used across the experiments in the study. Only male mice were used since $\mathrm{CPZ}$ toxicity effects the estrus cycle so that sex cannot be appropriately analyzed as a biological variable [36]. Statistical analysis and graphing was performed using GraphPad Prism software version 8.0 (RRID: SCR_002798). The values are shown as the mean \pm standard error of the mean (sem). Statistical 
significance was determined as $p<0.05$. The experimental design and statistical analysis for each section is provided below with the technical methods for that study.

\section{Mice and cuprizone model of demyelination}

Mice were housed and cared for in accordance with the National Institutes of Health Guide for the Care and Use of Laboratory Animals. The study protocol was approved by the Institutional Animal Care and Use Committee of the Uniformed Services University of the Health Sciences. Mice were socially housed in $27 \mathrm{~cm} \times 16.5 \mathrm{~cm} \times$ $12.5 \mathrm{~cm}$ cages (2-5 mice per cage) with enrichment objects and maintained on a standard $12 \mathrm{~h}$ cycle of daytime light (6:00-18:00). All procedures took place during the daytime light cycle.

C57BL/6 J mice (RRID:IMSR_JAX:000664, Jackson Laboratory, Bar Harbor, MA) at 8 weeks of age were fed pellets containing $0.3 \% \mathrm{CPZ}$ for 6 weeks or 12 weeks to produce acute or chronic demyelination, respectively. Milled CPZ powder (cat \#14690; Sigma-Aldrich; St. Louis, MO) was mixed as $0.3 \% \mathrm{CPZ}$ in normal chow (diet TD.01453; Harlan Teklad, Frederick, MD) to form pellets that were refreshed every 2-3 days. A dose of $0.3 \% \mathrm{CPZ}$ in pellets produces $\mathrm{CC}$ demyelination approximately equivalent to $0.2 \% \mathrm{CPZ}$ powder in ground chow [37-39]. Cohorts to be evaluated for remyelination received normal chow pellets for an additional 2 weeks after CPZ removal. Naïve mice continuously fed normal chow pellets served as a control non-demyelinated condition performed simultaneously along with $\mathrm{CPZ}$ treated mice. Baseline weights at the start of $\mathrm{CPZ}$ or normal chow feeding were similar within each cohort: MRI technical validation cohort (mean \pm sem $=$ naïve $25.25 \pm 0.47$ g; CPZ $25.25 \pm 0.43 \mathrm{~g}$ ), MRI iNSC transplant cohort (CPZ veh $26.24 \pm 0.42 \mathrm{~g} ; \mathrm{CPZ}$ iNSC $26.33 \pm 0.37 \mathrm{~g}$ ), wheels iNSC transplant cohort (naïve veh $22.87 \pm 0.40 \mathrm{~g}$; $\mathrm{CPZ}$ veh $23.76 \pm 0.56 \mathrm{~g}$; CPZ iNSC $23.76 \pm 0.61 \mathrm{~g}$ ). Mice remained in good health and regained baseline weights by the end of chronic $\mathrm{CPZ}$ administration.

\section{Magnetic resonance imaging (MRI)}

In vivo MRI was performed on a $7 \mathrm{~T}$ small animal $(20$ $\mathrm{cm}$ bore) Bruker BioSpec scanner equipped with $12 \mathrm{~cm}$ diameter $650 \mathrm{mT} / \mathrm{m}$ gradient coils (Bruker BioSpin $\mathrm{GmbH}$, Reinstetten, Germany). MRI with T2 weighting, magnetization transfer ratio (MTR), and diffusion tensor imaging (DTI) was used for longitudinal in vivo analysis of the effects of chronic CPZ ingestion and subsequent iNSC transplantation in the CC. Prior to image acquisition, mice were separated into yoked pairs of similar weight. During each image acquisition, mice were anaesthetized with $1 \%$ isoflurane. MRI slices were established using a sagittal localizer so that seven coronal slices were orientated perpendicular to the length of the $\mathrm{CC}$ and each scan was aligned with the midline crossing of the anterior commissure positioned in the same coronal slice [40-42].

For DTI [43], a three-dimensional (3D) single-shot echo planar imaging sequence (repetition time/echo time $[\mathrm{TR} / \mathrm{TE}]=900 / 36 \mathrm{msec} ; 1$ repetition) was used to acquire 4 unweighted $\left(\mathrm{b}=0 \mathrm{~s} / \mathrm{mm}^{2}\right)$ and 2 diffusionweighted images $\left(b=600,1200 \mathrm{~s} / \mathrm{mm}^{2}\right)$ in 14 noncollinear diffusion gradient directions using a Stejskal-Tanner diffusion preparation with parameters of $\Delta=12 \mathrm{msec}$ and $\delta=5 \mathrm{msec}$, field of view $(\mathrm{FOV})=14 \times 11.2 \mathrm{~mm}^{2}$, matrix $=80 \times 64 \times 24$, slice thickness $=750 \mu \mathrm{m}, 24$ slices, voxels $=175 \times 175 \times 750 \mu \mathrm{m}^{3}$. A whole brain $\mathrm{T} 2$ map was generated using a two-dimensional rapid acquisition with relaxation enhancement (2D RARE, corona [44]) with the following parameters: $\mathrm{TR}=4000 \mathrm{msec}$; $\mathrm{TE}=10$, 30, 50, 70, 90, $110 \mathrm{msec}$; RARE factor $=2$, number of averages $(\mathrm{NA})=4, \quad \mathrm{FOV}=14 \times 12 \mathrm{~mm}^{2}, \quad$ matrix $=112 \times$ $96 \times 18$, slice thickness $=750 \mu \mathrm{m}, 18$ slices, voxels $=$ $125 \times 125 \times 750 \mu \mathrm{m}^{3}$. For use in calculating the magnetization transfer ratio (MTR) [45, 46], 2D RARE sequence was used with $\mathrm{TR}=6000 \mathrm{msec}, \mathrm{TE}=20 \mathrm{msec}$, RARE factor $=8, \mathrm{NA}=4, \mathrm{FOV}=14 \times 12 \mathrm{~mm} 2$, matrix $=$ $112 \times 96 \times 18$, voxels $=125 \times 125 \times 750 \mu \mathrm{m}^{3}$, with saturation $(M s)$ and without $(\mathrm{Mo})$ using an offset saturation pulse ( $2 \mathrm{kHz}$ offset $66.7^{\circ}$ flip angle). Body temperature was maintained at $36^{\circ} \mathrm{C}$ by circulating hot water. Respiration and heart rate were monitored throughout each 2-h imaging session.

T2 and MTR maps along with T2 templates were generated from neuroimaging informatics technology initiative (NIFTI) files and analyzed in either VivoQuant (inviCRO, Boston, MA) (chronic CPZ) or MATLAB (MathWorks, Natick, MA)(chronic CPZ +2 weeks recovery). The CC ROI was defined as extending from the midline bilaterally to the point of ventral curvature in the external capsule, as in our previous studies (Sullivan et al. 2013 [47]; Yu et al. 2017 [42]). For each mouse, an initial ROI was propagated across images using the T2 templates and the voxel placement checked/corrected manually for each slice. The rostrocaudal extent of the $\mathrm{CC}$ was contained within 7 slices. Slices 1 and 7 were omitted to avoid regions of varying fiber directions toward the genu and splenium. Slices 2-6 (approximately $1 \mathrm{~mm}$ to $-2.5 \mathrm{~mm}$ relative to bregma) were combined as the full CC ROI. DTI data was analyzed with TORTOISE software [48, 49]. Diffusion-weighted imaging artifacts, including motion, eddy currents, and concomitant field distortions, were calculated and combined to enable the image correction to be applied in a single step. Fractional anisotropy (FA), Trace, and axial (AD) and radial (RD) diffusivity maps were computed using a nonlinear tensor estimation with RESTORE option [50]. Diffusion directionencoded color (DEC) maps were generated from the 
TORTOISE software to demonstrate fiber orientation. The CC ROIs were overlaid onto the DTI maps for further quantitative analysis.

\section{Technical validation for neuroimaging approach}

An MRI study with post-imaging neuropathology evaluated the MRI techniques for detection of chronic demyelination at the end of the chronic $\mathrm{CPZ}$ period, when iNSCs will be transplanted in the subsequent experiments. This longitudinal design compared naïve mice $(n=6)$ that received normal chow pellets continuously with CPZ treated mice $(n=6)$ at baseline (8 weeks of age), 6 weeks $\pm \mathrm{CPZ}$ (14 weeks of age), and 12 weeks \pm CPZ (20 weeks of age). Two-way ANOVA with repeated measures was used to compare naïve vs. $\mathrm{CPZ}$ at each time point, with Bonferroni multiple comparisons test.

\section{Neuroimaging analysis of iNSC transplantation effect on CC microstructure}

An MRI longitudinal study with post-imaging neuropathology evaluated the effect of iNSC transplantation on CC microstructure during recovery after chronic $\mathrm{CPZ}$. This longitudinal design compared CPZ treated mice injected with vehicle ( $n=5$, one mouse died) or iNSCs $(n=6)$. Scans were performed at baseline (8 weeks of age before starting CPZ), 6 weeks CPZ, 12 weeks CPZ, and then mice were returned to normal chow pellets until the final scan at 14 weeks. Mice received iNSC or vehicle intracerebral injections 1 day after the return to normal chow. Mice were scanned in sets of four per day, which were balanced by weights prior to the start of CPZ. After the week scan at 6 weeks of CPZ, mice within each set of four were randomly assigned, using Excel RAND, to receive iNSC or vehicle injection. The T2 values of the assigned groups were checked to ensure similar $\mathrm{CPZ}$ effect within the cohorts at 6 weeks. Two-way ANOVA with repeated measures was used for within subjects comparisons to baseline to evaluate $\mathrm{CPZ}$ pathology, and for comparison of each time point for iNSC vs. vehicle, with Bonferroni correction for multiple comparisons.

\section{Generation and characterization of mouse iNSCs}

Mouse C57BL/6 iNSCs that ubiquitously express farnesylated green fluorescent protein (GFP) were generated as previously detailed $[29,51]$. Briefly, iNSCs were produced by direct conversion of mouse embryonic fibroblasts through constitutive expression of Sox2, Klf4, cMyc, and transient expression of Oct4. iNSCs were then transduced with lentivirus containing a farnesylated GFP construct to target GFP to the inner plasma membrane. Prior to characterization and transplantation experiments, iNSCs were cultured as an adherent monolayer, as detailed previously $[29,51]$.

For in vitro characterization, iNSCs were cultured as either free floating neurospheres in proliferation medium or seeded onto glass coverslips coated with Matrigel ${ }^{\mathrm{Tm}}$ (Cat\# 356234 Corning, Corning, NY) for growth as adherent monolayers in differentiation medium, as previously detailed [51]. Differentiation medium contained NeuroCults basal medium (Cat\# 05700, STEMCELL Technologies, Cambridge, MA) with 10\% differentiation supplement (Cat\# 05703, STEMCELL Technologies) and 100 units penicillin $/ 0.1 \mathrm{mg}$ streptomycin $/ \mathrm{mL}$ (Cat\# 15140122 Invitrogen, Waltham, MA). For immunocytochemistry, neurosphere cultures were fixed on glass coverslips in 4\% paraformaldehyde (PFA, Sigma-Aldrich) $2 \%$ sucrose in $1 \mathrm{X}$ PBS. Neurospheres were incubated with primary antibodies for neural stem/progenitor cell markers Sox2 (rabbit polyclonal, 1:200; Abcam, Cambridge, MA, ab97959, RRID:AB_2341193) and Nestin (chicken polyclonal, 1:200; Abcam, ab134017, RRID:AB 2753197). Differentiation was examined using immunolabeling to detect markers of either the astroglial lineage (glial fibrillary acidic protein, GFAP; rabbit polyclonal, 1: 100; DAKO; Carpinteria, CA, Z0334, RRID:AB 10013382), the neuronal lineage (microtubule associated protein-2, MAP-2, rabbit polyclonal, 1:200; Abcam; ab32454, RRID:AB_776174), or the oligodendroglial lineage (O4 mouse monoclonal, 1:10 [52]). PBS with $0.1 \%$ Triton-X 100 and $10 \%$ normal goat serum for $1 \mathrm{~h}$ was used prior to all primary antibodies, but was not used with $\mathrm{O} 4$ to prevent Triton-X 100 degradation of the sulfatide epitope $[7,53]$. Secondary antibodies used were goat anti-rabbit IgG conjugated with AF647 (1:200; Thermo Fisher, Waltham, MA; A-21245, RRID:AB 2535813) to detect Sox2, and goat anti-chicken conjugated with AF555 (1:200; Thermo Fisher; A-21437, RRID:AB_2535858) to detect Nestin, donkey anti-rabbit IgG $\mathrm{F}(\mathrm{ab}) 2$ conjugated with Cy3 (1:400; Jackson ImmunoResearch, West Grove, PA, 711-166-152, RRID:AB 2313568) to detect GFAP, and goat anti-mouse IgM conjugated with Cy3 (1:50; Jackson ImmunoResearch; 115-166-075, RRID:AB_2338707).

\section{Technical validation for iNSCs preparations}

Frozen iNSCs were thawed and cultured for amplification and characterization for each transplantation experiment cohort. An aliquot of each iNSC preparation used for transplantation was analyzed to confirm neural stem cell characteristics. For differentiation analysis, the number of immunolabeled cells was counted in $n \geq 8$ nonoverlapping fields per sample up to a total of $n>300$ cells per aliquot. For growth curve analysis, the total number of cells was quantified after each passage of each subculture.

\section{Cell transplantation into the corpus callosum}

For all microinjections into the $\mathrm{CC}$, mice were anaesthetized with isoflurane (induction 3\%, maintenance 2\%) and a small $<1.0 \mathrm{~mm}$ diameter burr hole was drilled into 
the skull. A Hamilton gas tight syringe (Cat\# 7653-01; Hamilton Company, Reno, NV) was used with adapters (Cat\# 55750-01; Hamilton Company) and a pulled glass micropipette (outer diameter $50 \mu \mathrm{m}$ ) [54]. Each microinjection targeted the left $\mathrm{CC}$ using coordinates $(-1.0$ AP, 0.5 ML, $-1.3 \mathrm{DV}$ ) relative to bregma. An iNSC single cell suspension of $1 \times 10^{4}$ cells in $1 \mu \mathrm{l}$ of PBS, or PBS as a vehicle control, was injected over a 5 min period.

\section{Neurologic analysis with the miss-step running wheel assay} Starting the day after iNSC transplantation, and continuing for 2 weeks, mice were singly housed in home cages with a Miss-step running wheel and an optical sensor to detect wheel revolutions (Mouse Miss-step Activity Wheel system \#80821, Lafayette Instruments, Lafayette, IN). The Miss-step running wheels have 16 rungs missing from a standard wheel so that the remaining 22 rungs are distributed in an irregular interval pattern [33]. Whiskers were clipped so that the mice learn to locate each rung by bringing the hind paw forward to grasp the same rung as a forepaw [35]. Activity Wheel Monitor software (Lafayette Instruments) counted wheel revolutions at $6 \mathrm{~min}$ intervals during the light phase and 1 min intervals during the dark phase. Results were exported to a Microsoft Excel file every $24 \mathrm{~h}$.

\section{Technical validation for wheel running effects on oligodendrocyte populations in CC}

A study using acute $\mathrm{CPZ}$, which undergoes extensive spontaneous remyelination, tested the effect of the Missstep wheel exposure on the oligodendrocyte lineage response in the CC. Mice were fed normal chow (naïve, $n=6)$ or CPZ for 6 weeks to produce acute demyelination (acute CPZ, $n=12$ ). After 6 weeks, all mice were fed normal chow. The mice were then singly housed in home cages with (naïve, $n=6$, acute CPZ, $n=6$ ), or without (acute CPZ, $n=6$ ), Miss-step wheels for 2 weeks. Mice were perfused for in situ hybridization. Across the three conditions, cell densities were quantified from at least 3 sections per mouse and analyzed using one-way ANOVA with Tukey's multiple comparisons test.

\section{Miss-step wheel analysis of iNSC transplantation on CC function}

Running on Miss-step wheels, with irregularly spaced rungs, presented a novel motor skill task to evaluate effects of iNSC transplantation on sensorimotor function during recovery after chronic CPZ. Cohorts were maintained under the same conditions for simultaneous automated data collection. The study included four cohorts of 12 mice each, which included naïve with vehicle injection $(n=4), \mathrm{CPZ}$ with vehicle injection $(n=4)$, and CPZ with iNSC transplant $(n=4)$. Of the four cohorts run, one cohort was excluded due to the $\mathrm{CPZ}$ vehicle condition failing to demonstrate a demyelination associated deficit, compared to naïve. Data from 3 independent cohorts of 12 mice each was combined for analysis of naive $(n=11)$, CPZ vehicle $(n=12)$, and CPZ iNSC $(n=11)$. Death prior to the experimental endpoint required exclusion of one mouse (CPZ iNSC, $n=1$ ). A mouse (naive, $n=1$ ) that did not achieve criterion (average velocity of $10 \mathrm{~m} / \mathrm{min}$ by day 7) during the learning phase was excluded from analysis of the plateau phase. A value missing due to technical errors of the wheel system was imputed based on the mean ( $n=1$ mouse on $1 \mathrm{~d})$. Velocity measures were compared using repeated measures two-way ANOVA with Sidak's multiple comparison test. For in situ hybridization analysis in tissues from mice with and without wheel exposure, additional mice (naive veh, $n=5$; CPZ veh, $n=5$ ) were housed singly without wheels in parallel with the wheel mice from two different cohorts.

Tissue analysis of neuropathology and cellular responses After completion of imaging or Miss-step wheel assessments, mice were perfused for tissue analysis. Imaging and wheel running cohorts were separately analyzed. Quantification included at least 3 tissue sections per mouse, unless otherwise noted. The number of mice for each condition is provided with each experiment above and is stated in each figure legend. Images for quantification were acquired using an Olympus IX70 fluorescence microscope with a SPOT RT3 camera (Diagnostic Instruments, Sterling Heights, MI). The CC ROI was defined as extending from the midline bilaterally to the point of ventral curvature in the external capsule [42, 47]. Coronal sections within -0.5 and $-2.0 \mathrm{~mm}$ relative to bregma were used for quantification, to focus in the most demyelinated region and minimize variation [40]. Unpaired $t$-tests were used to compare two conditions, i.e. naïve vs. CPZ, or veh vs. iNSCs. After the Miss-step wheel running, further analysis of oligodendrocyte populations for $\mathrm{CPZ}$ veh vs. CPZ iNSCs used two-way ANOVA with Sidak's multiple comparisons test.

\section{Immunohistochemistry}

Mice were perfused transcardially with $4 \%$ PFA, postfixed overnight at $4{ }^{\circ} \mathrm{C}$, and processed for frozen sectioning. Coronal cryosections $(15 \mu \mathrm{m})$ were immunostained with primary antibodies to detect either myelin based on myelin oligodendrocyte glycoprotein (MOG, mouse monoclonal, 1:100; EMD Millipore, Burlington, MA; mab5680, RRID:AB_1587278), astrocytes with GFAP (rabbit polyclonal, 1:500; DAKO; Z0334, RRID:AB_ 10013382), microglia/macrophage with IBA1 (rabbit polyclonal, 1:500; Wako, Richmond, VA; 019-19,741, RRID:AB_839504), oligodendrocyte lineage cells with 
Olig2 (rabbit polyclonal, 1:500; EMD Millipore, ab9610, RRID:AB_570666), OPCs with NG2 (rabbit polyclonal to ectodomain; generous gift from William Stallcup; RRID: AB_2572306) and neural stem/progenitor cells with Sox2 (rabbit polyclonal, 1:200; Abcam, ab97959, RRID: AB_2341193). Cells undergoing active proliferation were identified by Ki67 (rabbit polyclonal, 1:500; Abcam ab 15,580, RRID:AB_443209). Axons were identified by neurofilament heavy chain protein $(\mathrm{NF}-\mathrm{H})$ rabbit polyclonal antibody (1:500; RPCA-NF-H Encor Biotechnology, Gainesville, FL, RRID:AB_2572360) with the proportion with axon damage immunolabeled for SMI32 mouse IgG1 monoclonal antibody, which recognizes nonphosphorylated neurofilaments (1:1000; Sternberger monoclonal SMI32; 801,701 Biolegend, San Diego, CA, RRID:AB_2564642). The secondary antibodies used were donkey anti-rabbit IgG $\mathrm{F}(\mathrm{ab}$ ')2 (Jackson ImmunoResearch, West Grove, PA) conjugated with Cy3 (1:400 or 1:500; 711-166-152, RRID:AB_2313568) or AF647 (1: 100; 711-606-152, RRID:AB_2340625), donkey antimouse IgG IgG $\mathrm{F}\left(\mathrm{ab}^{\prime}\right) 2$ conjugated with Cy3 (1:100; Jackson ImmunoResearch; 715-166-150, RRID:AB 2340816), goat anti-rabbit IgG conjugated with AF647 (1:200; Thermo Fisher; A21245, RRID:AB_2535813) and goat anti-mouse IgG conjugated with AF555 (1:500; Thermo Fisher; A21422, RRID:AB_2535844). Nuclei in all sections were counterstained with DAPI (Sigma-Aldrich). The GFP expression of iNSCs was sufficient for direct detection without using additional antibody detection.

\section{In situ hybridization}

In situ hybridization using a proteolipid protein (Plp1) riboprobe was performed as previously described [55, 56]. The Enpp6 plasmid vector (pCMV-SPORT6, gift from Dr. William D. Richardson, University College London) was used to generate the Enpp6 riboprobe (Xiao et al. 2016 [57]). In $15 \mu \mathrm{m}$ coronal cryosections, hybridized Plp1 or Enpp6 riboprobe was detected with alkaline phosphatase-conjugated sheep anti-digoxigenin antibody and incubation in substrate solution (nitroblue tetrazolium chloride/5-bromo-4-chloro-3-indolyl-phosphate [NBT/BCIP]; Dako).

\section{Quantification details for CC area, myelin, astrogliosis and microglia activation}

Immunolabeling within the CC ROI was quantified on images acquired with a 10x objective. Metamorph software (RRID:SCR_002368; Molecular Devices, Downington, PA) was used to measure the total CC ROI area in coronal sections immunolabeled for MOG along with DAPI staining of nuclei for cytoarchitecture of $\mathrm{CC}$ as distinct from adjacent regions. Myelination of the CC was measured based on pixel intensity values to determine the MOG immunolabeled pixels above background levels using the Metamorph thresholding function [20]. Similar thresholding was used to quantify astrogliosis and microglia activation based on GFAP and IBA1 immunoreactivity, respectively.

\section{Quantification details for structure tensor analysis of astrocytes and myelin}

NIH ImageJ software (ImageJ, RRID:SCR_003070) with the OrientationJ Plug-in (RRID:SCR_014796, http:// bigwww.epfl.ch/demo/orientation/) was used for structure tensor analysis [58]. Images were acquired with a $10 x$ objective. Using the polygon tool, the ROI was selected within the $\mathrm{CC}$ under the medial extension of the cingulum. This $\mathrm{CC}$ region avoids the curvature toward the midline and the crossing fibers that are present more laterally. The program computes the microscopic, or local, orientation and local coherence for each pixel. The local orientation uses a color map to represent the directional distribution. The local coherence is a measure of the alignment of anisotropic domain tensors. Both the anisotropy of a local domain and the coherence of domains within a voxel contribute to fractional anisotropy [59].

\section{Quantification details for oligodendrocyte lineage populations}

Oligodendrocyte counts in the CC were based on in situ hybridization and quantified from bright field images with the CC ROI area measured using Spot Advanced Software (RRID: SCR_014313; Spot imaging solutions, Sterling Heights, MI). Plp1 $1^{\text {low }}$ expressing cells had mRNA transcripts localized mainly in the perinuclear cytoplasm; in Plp $1^{\text {high }}$ expressing cells, darker substrate reaction was evident in the cell body and extended out into processes $[20,47,60]$. Only cells with strong substrate reaction for Enpp6 transcript levels were counted as specific labeling of newly formed oligodendrocytes [57]. Quantification of proliferating OPCs in the CC and cingulum were identified based on Ki67 immunoreactive nuclei and NG2 immunolabeling of the cell body and processes. Ki67 and NG2 analysis included only one section per mouse due to the limited availability of tissue within the defined coronal levels.

\section{Quantification details for axon damage}

Confocal images were acquired at $63 \mathrm{x}$ and quantified in maximum intensity projections of the ROI $(59.70 \mu \mathrm{m}$, y: $59.70 \mu \mathrm{m}, \mathrm{z}: 1.60 \mu \mathrm{m})$ in the cingulum. The ROI was positioned adjacent to the $\mathrm{CC}$ and centered under the peak of the cingulum. Individual axons were manually counted as immunolabeled for NF-H with or without co-labeling for SMI32. Nuclei were counted simultaneously. Ipsilateral 
and contralateral sides were quantified in at least $3 \mathrm{sec}-$ tions per mouse.

\section{Transplanted iNSC localization and differentiation in vivo} Transplanted iNSCs were quantified by direct visualization of GFP expression using a 40x objective on an Olympus IX-70 microscope. Tissue sections were analyzed from mice in the imaging $(n=6)$ and Miss-step wheel assessments $(n=11)$ that were used for quantification of MOG, GFAP, and IBA1 immunoreactivity. The in situ hybridization reaction for Plp1 precluded identification of GFP expression from iNSCs. Additional tissue sections were immunostained for labeling of iNSCs with cell type markers. Overall, this iNSC cell type quantification included at least 6 mice per cell type immunostain with at least 3 sections analyzed per mouse combining to approximately 200 iNSCs each for Sox 2 and for Olig2, with approximately 600 iNSCs counted for GFAP which included sections from the neuropathology analysis of astrogliosis.

Transplanted iNSCs were analyzed in vivo only within coronal sections from rostrocaudal levels matching the neuropathology ROI $(-0.5 \mathrm{~mm}$ to $-2.0 \mathrm{~mm}$ from bregma), which focused on the most demyelinated CC region and minimize variation [40]. Regions of the cerebral cortex and cingulum above the $\mathrm{CC}$, and hippocampus below the $C C$, were quantified within the same coronal sections as the CC. In total, 1585 iNSC cells were counted from among mice combined from MRI and Miss-step wheel cohorts and graphed to represent the distribution within the tissue as white matter (CC, cingulum) and adjacent gray matter (cortex, hippocampus). Contingency tables were used to analyze in vivo differentiation of iNSCs after transplantation with Fisher's exact test for statistical significance.

Additional images of GFP cells were acquired on a Zeiss 700 laser scanning confocal microscope (Carl Zeiss, San Diego, California; RRID:SCR_017377) with individual laser lines sequentially collected for each channel using a Plan-Apochromat $63 x / 1.4$ oil objective. An optical image stack was acquired, and maximum intensity projection images were generated from each image stack with Zen software (Carl Zeiss, ZEN Digital Imaging for Light Microscopy, RRID:SCR_013672).

\section{Results}

\section{Characterization of iNSC preparations used for transplantation}

Induced neural stem cells (iNSCs) have the potential to self-renew and to differentiate along three neural lineages $[29,31,32,51]$. Following revival and expansion, iNSCs were assessed for self-renewal as neurospheres (Fig. 1a-b) and differentiation along neural lineages (Fig. 1c-e). In vitro, iNSCs demonstrated immunolabeling for neural stem/progenitor cell markers, Nestin and Sox2, and neurosphere formation (Fig. 1a-b). iNSC self-renewal was exhibited by $\log$ phase cell amplification after each passage (Fig. 1f). Following growth in differentiation media, iNSCs acquired markers for either astroglial (GFAP, Fig. 1c), neuronal (MAP2, Fig. 1d), or oligodendroglial (O4, Fig. 1e) lineages. Differentiation was confirmed for each aliquot of iNSCs used for transplantation experiments (Fig. 1g).

\section{Longitudinal MRI technique evaluation with post-imaging validation of chronic CPZ pathology}

Prior to studies of iNSC transplantation, we first tested the set of MRI techniques for quantification of the progression of CPZ acute and chronic demyelination (Fig. 2). These techniques quantitatively demonstrate MRI changes in the $\mathrm{CC}$ at 6 and 12 weeks after CPZ in comparison with naïve mice fed normal chow across the same time course (Fig. 2a). The T2 and direction-encoded color images show that acute and chronic CPZ changes are most marked in the medial CC (Fig. 2b). CPZ increased the T2weighted signal, which reflects increased unbound water, while reducing the magnetization transfer ratio (MTR) associated with water bound to membranes, including myelin (Fig. 2c-d). Diffusion tensor imaging (DTI) values provided complementary measures (Fig. 2e-h). Trace values indicated increased water diffusion within the $\mathrm{CC}$ after CPZ. Reduced fractional anisotropy (FA) was driven by increased radial diffusivity (RD), which can be associated with demyelination. Axial diffusivity (AD) was not altered after 6 or 12 weeks of CPZ, which is in agreement with our prior studies that found axonal beading and altered $\mathrm{AD}$ at 3-4 weeks of $\mathrm{CPZ}$ was attenuated by the 6 week time point $[40,41,61,62]$.

Post-imaging neuropathology focused on CC myelination and neuroinflammation to characterize the chronic lesion environment into which iNSCs would be transplanted in subsequent experiments (Fig. 3). Mice from the MRI cohort were perfused after the final scan. Chronic CPZ mice had significant CC atrophy, based on the reduced area measured, and significant demyelination demonstrated with immunolabeling of myelin. Chronic CPZ also resulted in persistent astrogliosis, which is a feature of sclerotic lesions. Increased microglial signal also indicated CC neuroinflammation.

\section{Longitudinal MRI and post-imaging neuropathology changes after iNSC transplantation during chronic demyelination}

Longitudinal MRI scans of each mouse at multiple time points were used to demonstrate the progression of $\mathrm{CPZ}$ pathology before, and then after, iNSC or vehicle injection (Fig. 4a). Representative T2 and direction-encoded color images illustrate baseline, i.e. before initiating CPZ, for comparison with the final scan after $\mathrm{CPZ}$ and either 

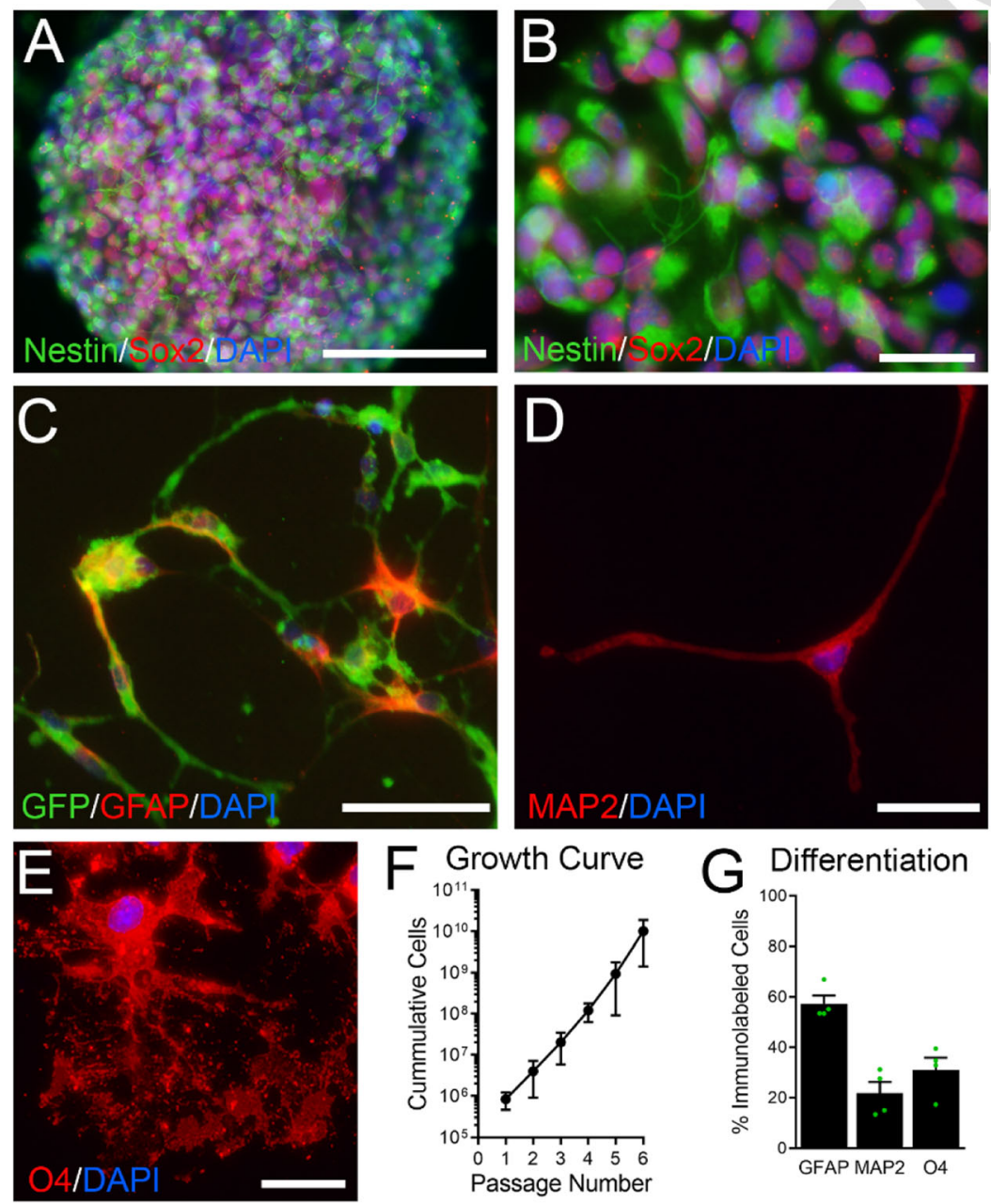

Fig. 1 Mouse induced neural stem cells (iNSCs) exhibit stem cell characteristics in vitro. a-b: In vitro, iNSCs form neurospheres (a) and express NSC markers Sox2 (red) and Nestin (pseudocolored green). c-e: Following differentiation of iNSCs, constitutively expressed green fluorescent protein (GFP) is visible in all cells (c) while cells differentiated along an astrocytic lineage exhibit immunolabeling for GFAP (red, c). In addition, differentiation along neuronal (MAP2; red, $\mathbf{d}$ ) and oligodendroglial (O4; red, e) lineages shows multipotency of iNSCs (GFP not shown). f: In nondifferentiation conditions, iNSCs exhibit an exponential proliferation rate expected for stem cell growth based on quantification of each subculture after each passage. g: Quantification of iNSC multipotent differentiation from separately revived vials $(n=4)$. Data are mean values \pm sem. Scale bar $A=100 \mu m, B, D, E=20 \mu m, C=40 \mu m$

vehicle or iNSC injection (Fig. 4b). Robust reproducibility is indicated by the similar changes observed from baseline to 6 and 12 week CPZ time points in this cohort (Fig. 4c-h) relative to naïve mice and baseline vs. 6 and 12 week CPZ time points in the validation cohort (Fig. 2c-h). At 12 weeks of $\mathrm{CPZ}$, values are increased for T2, Trace and RD; CPZ decreased MTR and FA, while AD was not changed at 12 weeks (Fig. 4c-h). After removal of $\mathrm{CPZ}$ and return to normal chow, mice received iNSC or vehicle injection into the CC. Notably, the iNSC transplants resulted in significant recovery of DTI RD values (Fig. 4h), suggesting an iNSC effect on CC microstrucuture that did not significantly change the other imaging parameters (Fig. 4c-g).
Post-imaging neuropathology focused on CC myelination and neuroinflammation, with additional analysis of the endogenous oligodendrocyte population after iNSC transplantation (Fig. 5). Mice that received iNSCs did not exhibit overt changes in CC myelination, as compared to vehicle-injected mice (Fig. 5a-b). Transplanted iNSCs were found in the $\mathrm{CC}$ and the overlying white matter of the cingulum, and in adjacent gray matter of the adjacent cortex and hippocampus (Fig. 5c). The iNSCs exhibited mainly rounded morphologies, while some cells had extended processes (Fig. 5d). To determine whether transplanted iNSCs elicited a response among endogenous CC cells, sections were examined using immunolabeling for GFAP for astrocytes (Fig. 5c-d), IBA1 for microglia (Fig. 5e), and 

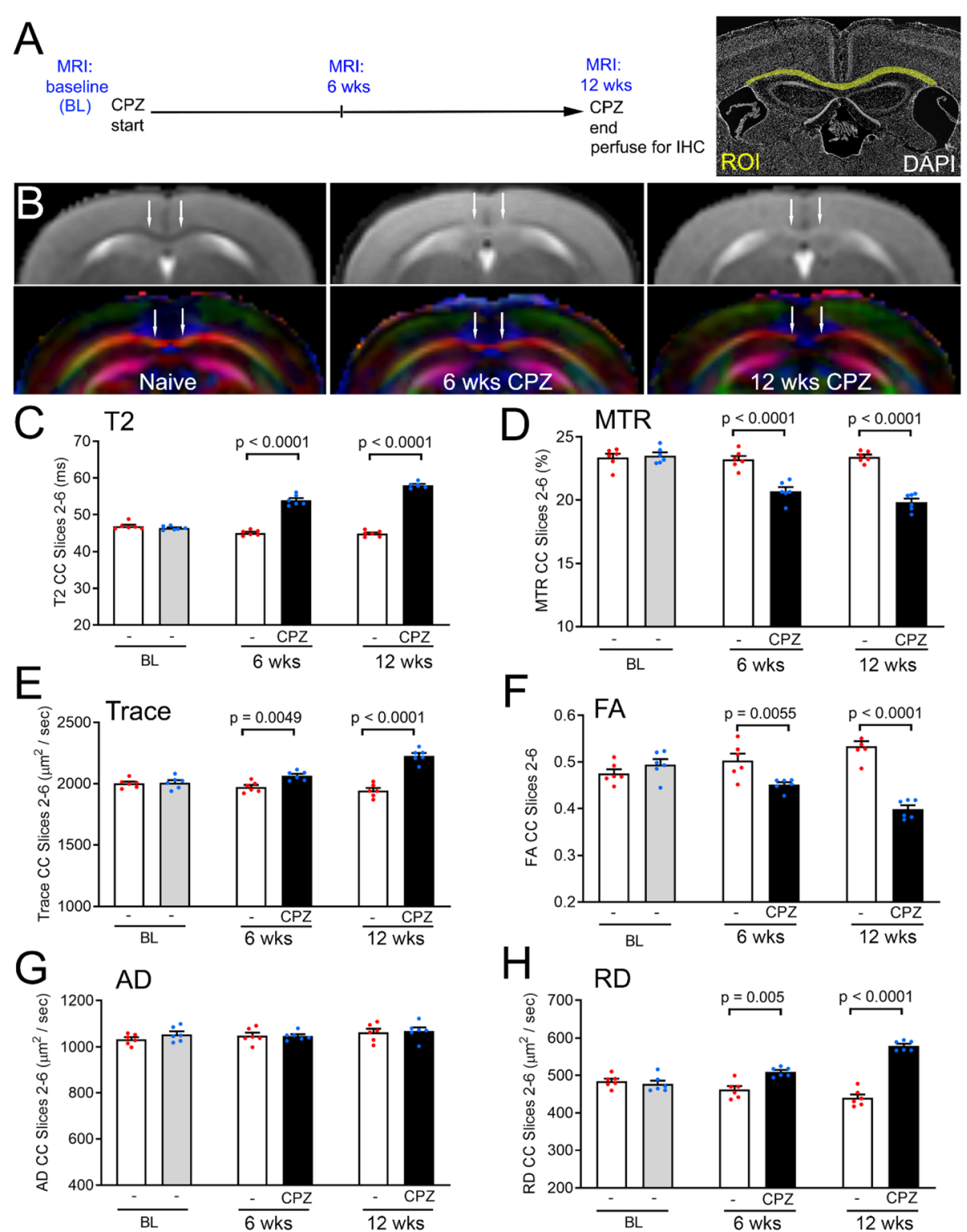

Fig. 2 MRI techniques detect progression of corpus callosum (CC) pathology during acute through chronic cuprizone (CPZ). a: Experimental timeline for longitudinal in vivo magnetic resonance imaging (MRI) of the same mice scanned three times: at baseline (BL, 8 weeks of age prior to the start of CPZ), again after acute CPZ (6 weeks) and finally after chronic CPZ (12 weeks). Naïve mice were scanned at matched time points but continuously fed normal chow pellets without CPZ. CC region-of-interest (ROI) for quantification shown in yellow with DAPI nuclear staining of cytoarchitecture. b: Representative T2-weighted images with corresponding direction encoded (DEC) color images below for each set. T2 panels show increased signal intensity in the CC during acute and chronic CPZ while DEC maps show altered CC microstructure, as compared to naïve mice (age-matched to the 12 week CPZ time point). Arrows indicate medial CC regions that show CPZ effect. Colors represent fiber directions as red (medial-lateral), blue (anterior-posterior), and green (superior-inferior). c-h: Quantitative analysis of the CC shows significant differences between naive and CPZ mice at both 6 weeks and 12 weeks after the start of CPZ using values from T2-weighted imaging (c), magnetization transfer ratio (MTR, d), and diffusion tensor imaging parameters of trace (e), fractional anisotropy (FA; $\mathbf{f})$, and radial diffusivity (RD; $\mathbf{h})$. Axial diffusivity (AD, $\mathbf{g}$ ) remained unchanged. Bar color reflects the treatment condition. The naïve cohort (white; no cuprizone shown as "-"under bars) was scanned along with the CPZ cohort at each of the three time points. The CPZ cohort was scanned at baseline (gray; "-"before starting CPZ) and during demyelination (black; at 6 and 12 weeks of CPZ). Data are mean values \pm sem. Two-way ANOVA with repeated measures and Bonferroni multiple comparisons test for naïve $(n=6)$ versus CPZ $(n=6)$ mice

in situ hybridization to detect oligodendrocyte expression of Plp1 (Fig. 5f). The findings of CC atrophy and demyelination at 12 weeks of CPZ (Fig. 3g-h) persisted at similar levels after the 2-week recovery period on normal chow, and were not attenuated by iNSC transplantation (Fig. $5 \mathrm{~g}$ h). Interestingly, astrogliosis persisted in vehicle-injected 

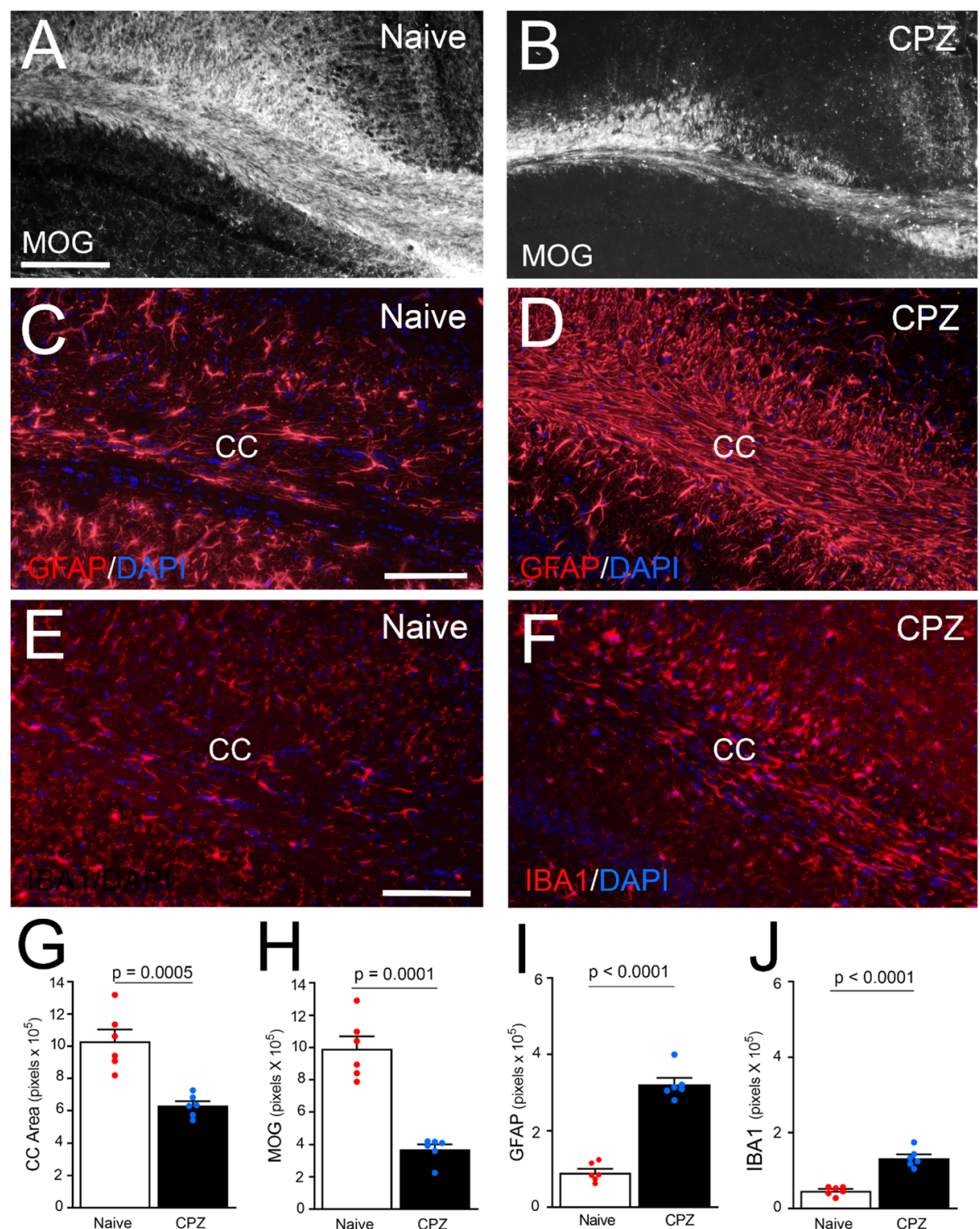

Fig. 3 Post-MRI pathology after chronic CPZ. a-f: Coronal sections from naïve and 12 wk. CPZ perfused after final MRI (Fig. 2) and immunolabeled for either myelin (MOG; a-b), astrocytes (GFAP; $\mathbf{c}$-d), or microglia (IBA1; e-f), along with DAPI nuclear stain (blue; c-f). g-j: Quantification of immunolabeling in this post-MRI cohort shows that chronic CPZ results in significant CC atrophy $(\mathbf{g})$ with demyelination (h), astrogliosis (i), and microglial activation (j). CC area was measured using DAPI nuclear staining of cytoarchitecture and MOG immunoreactivity. Bar color reflects condition as naïve (white; no cuprizone), or demyelinated (black; 12 weeks of cuprizone). Data are mean values \pm sem. Naïve $(n=6)$ compared to chronic CPZ $(n=6)$ using $t$-tests. Scale bars A, B: shown in $A=200 \mu m$; C, D: shown in C = $100 \mu m$; E, F: shown in $E=100 \mu m$

mice and was significantly reduced with iNSC transplantation (Fig. 5i). The microglial immunoreactivity did not show an effect of iNSC transplantation (Fig. 5j). The oligodendrocyte population appeared only slightly increased after iNSC transplantation, and did not reach a statistically significant effect (Fig. 5k).

The astrogliosis is a remarkable feature of this chronic demyelination model (Figs. 3c-d, 5c-d). Given the high astrocyte density and aligned orientation, we examined the structure tensor characteristics of CC immunolabeled astrocytes after chronic CPZ (Fig. 6). Structure tensor analysis applied to histological tissue can quantify microscopic features that add to the interpretation of tissue microstructure, and has been used for analysis of myelin immunolabeling in chronic CPZ [58, 59, 63]. The images used to quantify GFAP immunoreactivity post-MRI (Figs. $3 i, 5 i)$ were used for structure tensor analysis. In naïve mice, GFAP immunolabeled cells had a relatively isotropic appearance (Fig. 6a). After chronic CPZ, the GFAP immunolabeled cells exhibited coherence of local domains for 

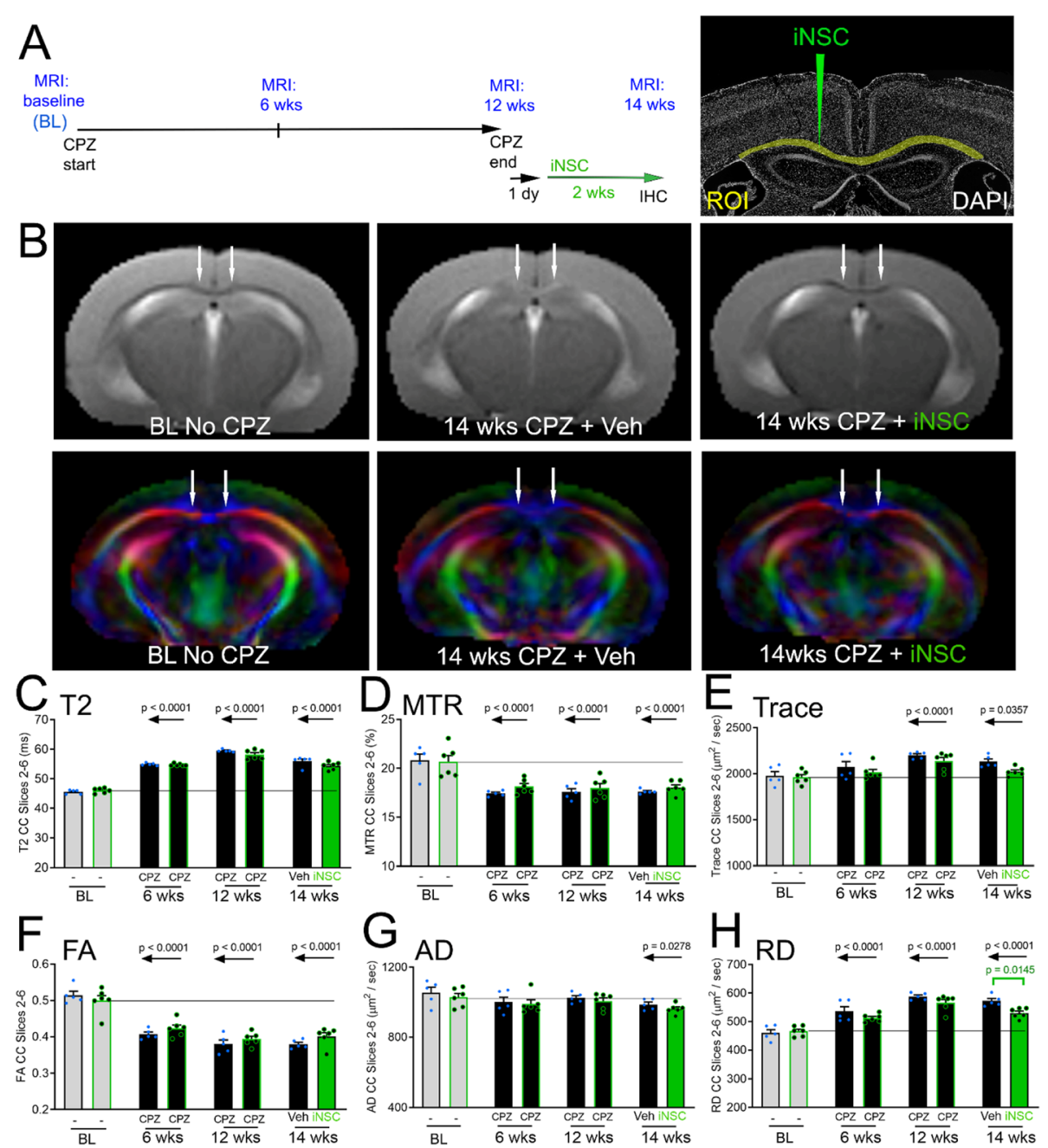

Fig. $4 \mathrm{MRI}$ radial diffusivity indicates reduced CC pathology with iNSC transplantation. a: Experimental timeline for longitudinal imaging of mice across 4 time points: baseline (BL) before initiating CPZ, at 6 weeks of CPZ, and at 12 weeks of CPZ before and finally at 2 weeks after iNSC, or vehicle, injection. After the scan at 6 weeks of CPZ, mice were randomized to vehicle or iNSC injection. After 12 weeks of CPZ, mice were returned to normal chow pellets for 1 day before intracerebral injection. CC region-of-interest (ROI) for quantification shown in yellow with iNSC injection path shown in green, with DAPI nuclear staining of cytoarchitecture. b: Representative T2-weighted and DEC images. Arrows indicate medial CC regions that show CPZ effect. Colors reflect fiber directions as red (medial-lateral), blue (anterior-posterior), and green (superior-inferior). c-h: Quantitative analysis of the CC shows significant differences from baseline result from CPZ administration using values from T2-weighted imaging $(\mathbf{c})$, MTR $(\mathbf{d})$, trace $(\mathbf{e})$, FA (f), and RD $(\mathbf{h})$. The AD values only differ from baseline at the 14 week time point (g). The comparison of iNSC transplantation versus vehicle (Veh) shows a significant effect in RD values (h, green bracket). Horizontal line shown extends from the baseline value of the cohort that later received iNSCS after 12 weeks of CPZ. Bar color reflects condition as baseline (gray fill), demyelinated with CPZ (black fill) or iNSC transplant after chronic CPZ (green fill). Borders around bars denote same mice that received iNSC (green border) or vehicle (black border) after ending cuprizone. Data are mean values \pm sem. Two-way ANOVA with repeated measures and Bonferroni multiple comparisons test for CPZ with injection of vehicle $(n=5)$ versus iNSCs $(n=6)$

effective anisotropy (Fig. 6b). In naïve mice, astrocyte processes extended in many directions, as indicated by varied color encoding of the orientation (Fig. 6c). After CPZ, reactive astrocytes were highly oriented in parallel to the axis of axons, as indicated by the mainly green encoded orientation in the CC but magenta in the perpendicularly oriented cingulum tract (Fig. 6d). Microscopic, or local, GFAP coherence was significantly increased within the $\mathrm{CC}$ after 12 weeks of CPZ compared to naïve mice (Fig. 6e). Surprisingly, the GFAP coherence level was as high as that measured for MOG immunolabeling of myelin (Fig. 6f, g). Myelin and axons are considered major components influencing diffusion tensor imaging values, although cell density and edema are also important factors [64]. These results raise the possibility that astrogliosis can significantly contribute to microstructure signal, which may be relevant for sclerotic MS lesions. Reduced overall GFAP immunolabeling after iNSC transplantation (Fig. 5i) may 

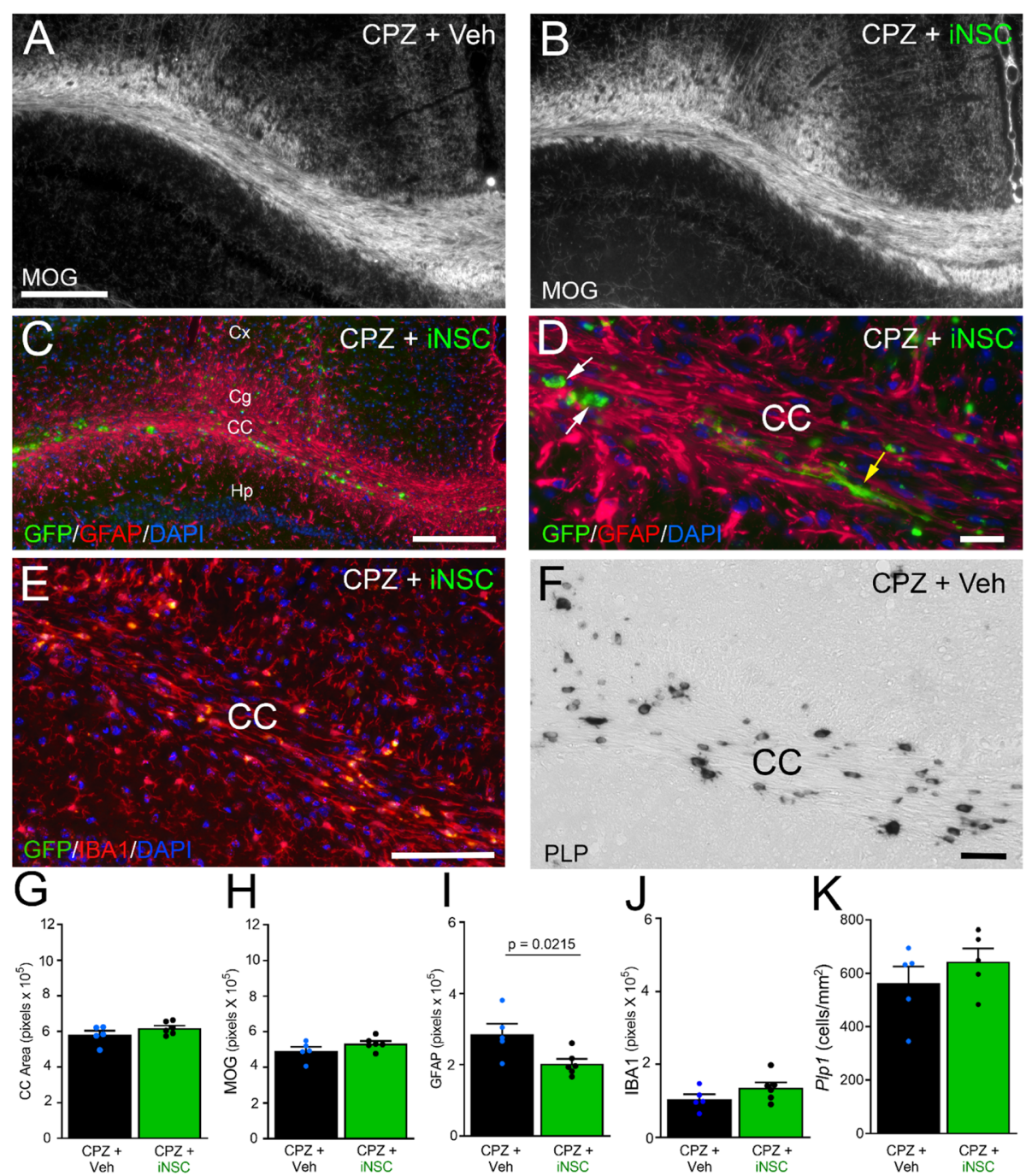

Fig. 5 Post-MRI pathology shows iNSC reduction of astrogliosis. a-e: Coronal sections from chronic CPZ mice with vehicle (Veh) or iNSC injection immunolabeled to detect myelin (MOG; $\mathbf{a}-\mathbf{b}$ ), or the innate immune response of astrocytes (GFAP; $\mathbf{c}-\mathbf{d}$ ) or microglia (IBA1; e) with DAPI nuclear stain (blue; c-e). Panel C shows an example of transplanted iNSCs (green, GFP) in the CC and adjacent white matter (cingulum; Cg) and gray matter regions (cortex, Cx; hippocampus, Hp). Panel D shows higher magnification of iNSCs (green, GFP) in the CC with examples of round cells (white arrows) and elongated cells with extended processes (yellow arrow). Panel E shows IBA1 immunolabeling of microglia (red) with lipofuscin granules (yellow autofluorescence) that accumulate after chronic CPZ. f: Coronal CC sections processed for in situ hybridization to detect oligodendrocytes expressing proteolipid protein (PIp1). g-k: Quantification of tissue analysis in this post-MRI cohort shows CC atrophy and demyelination are not attenuated by iNSCs $(\mathbf{g}, \mathbf{h})$. Transplanted iNSCs reduced astrogliosis (i), but did not change the microglial $(\mathbf{j})$ or oligodendrocyte response (k). Bar color reflects condition as chronic cuprizone followed by vehicle injection (black) or iNSC transplant (green). Data are mean values \pm sem. Vehicle $(n=5)$ and iNSC $(n=6 \mathrm{HHC}, n=5 \mathrm{Plp} 1)$ conditions were compared using $t$-tests. Scale bars: A, B shown in $A=200 \mu m ; C=200 \mu m ; D=20 \mu m ; E=100 \mu m ; F=40 \mu m$

reduce the overall anisotropic contribution of reactive astrocytes among all the cellular structures contributing to the DTI FA. The DTI AD component of FA is strongly driven by axons [61], and was not significantly different after iNSC transplantation (Fig. 4g). Conversely, the DTI $\mathrm{RD}$ component can be driven by myelin changes [61]. Our data suggest that the reduced DTI RD after iNSC transplantation (Fig. 4h) may possibly be influenced by changes in reactive astrogliosis after chronic CPZ, since GFAP and MOG immunoreactivity exhibited similar coherence (Fig. 6f-g). However, our 2-dimensional pixel scale structure tensor analysis can reveal local microstructure features but cannot demonstrate specific correlation to the much larger 3-dimensional DTI voxel scale features.

\section{Reduced neurologic impairment after iNSC transplantation during chronic demyelination}

We next tested whether iNSC transplantation could reduce neurologic functional deficits after chronic $\mathrm{CPZ}$ (Fig. 7). Running on Miss-step wheels, with irregularly 

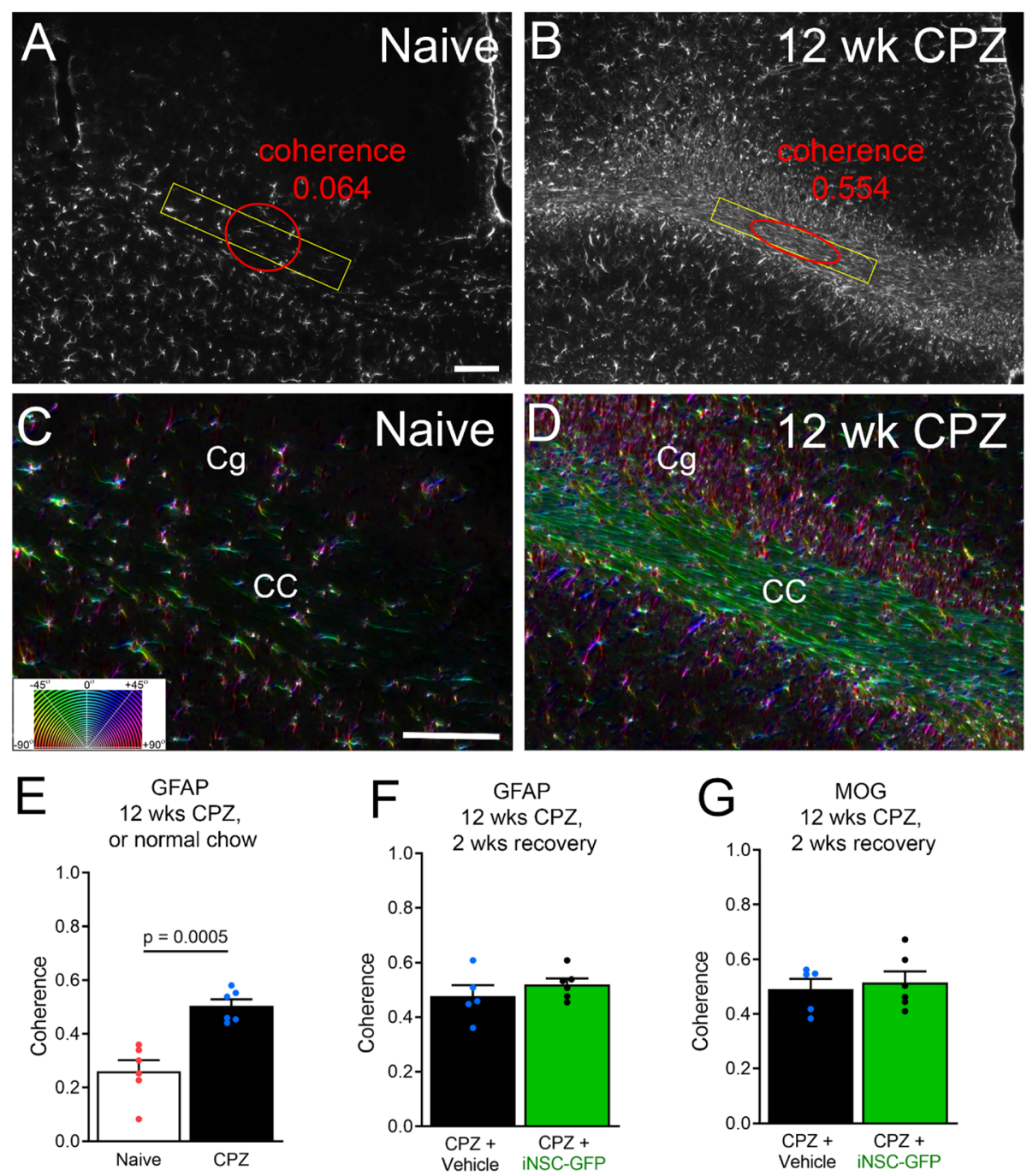

Fig. 6 Astrogliosis of chronic CPZ increases coherence within CC. a-b: Coronal sections from naïve and 12 week CPZ mice perfused after final MRI and immunolabeled for GFAP to detect astrocytes. Coherence of astrocyte morphology was measured in a CC area (yellow rectangle) of relatively aligned anatomical structure. In the non-pathological CC of naive mice, the astrocyte density is low and cell processes extend in many directions, resulting in close to isotropic coherence $(\mathbf{a}$; red). After $\mathrm{CPZ}$, astrocytes have a high density and align parallel to axons in the chronically demyelinated CC, so that the GFAP immunoreactivity exhibits anisotropic coherence (b, red). c-d: Color maps illustrating the orientation (c, inset) of the astrocyte cell bodies and processes in higher magnification images taken from the same sections as in A and B. In naïve mice, astrocyte cell bodies and processes exhibit diverse color encoding indicating orientation in many directions (c). After CPZ, astrocytes are aligned parallel to axons in the CC (green color orientation) and in the perpendicularly aligned cingulum (Cg) tract (magenta color orientation). e-g: Quantification of the coherence shows a significant increase in CPZ demyelinated mice (e) that does not recover during the 2 weeks on normal chow ( $\mathbf{f}$ ), and is not changed in iNSC transplant mice (f). In sections immunolabeled for MOG ( $\mathbf{g}$ ), the coherence for myelin is similar to that measured for astrocytes (f-g). Bar color reflects condition as chronic cuprizone followed by vehicle injection (black) or iNSC transplant (green). Data are mean values \pm sem. Mouse numbers shown in Figs. 3 and 5, with comparison by $t$-test. Scale bars A, B shown in $A=100 \mu m ; C, D$ shown in C = $100 \mu m$

spaced rungs, was used because this assessment is sensitive to deficits associated with $\mathrm{CPZ}$ demyelination and targeted to CC function [33, 57]. After 12 weeks of CPZ, mice were returned to normal chow and then received iNSC or vehicle injections followed by exposure to Missstep wheels for 2 weeks (Fig. 7a). Age matched naïve mice received vehicle injections and were maintained within the $\mathrm{CPZ}$ cohorts. Comparison of vehicle-injected controls showed significant deficits between naïve and chronic CPZ, which were evident in both the average daily running velocity (Fig. $7 \mathrm{~b}$ ) and the maximal daily running velocity (Fig. 7c). Chronic CPZ impaired motor skill learning (learning phase) with significant performance deficits (plateau phase). On the contrary, mice with iNSC transplantation did not have significant deficits in either the average or maximum running velocities after 

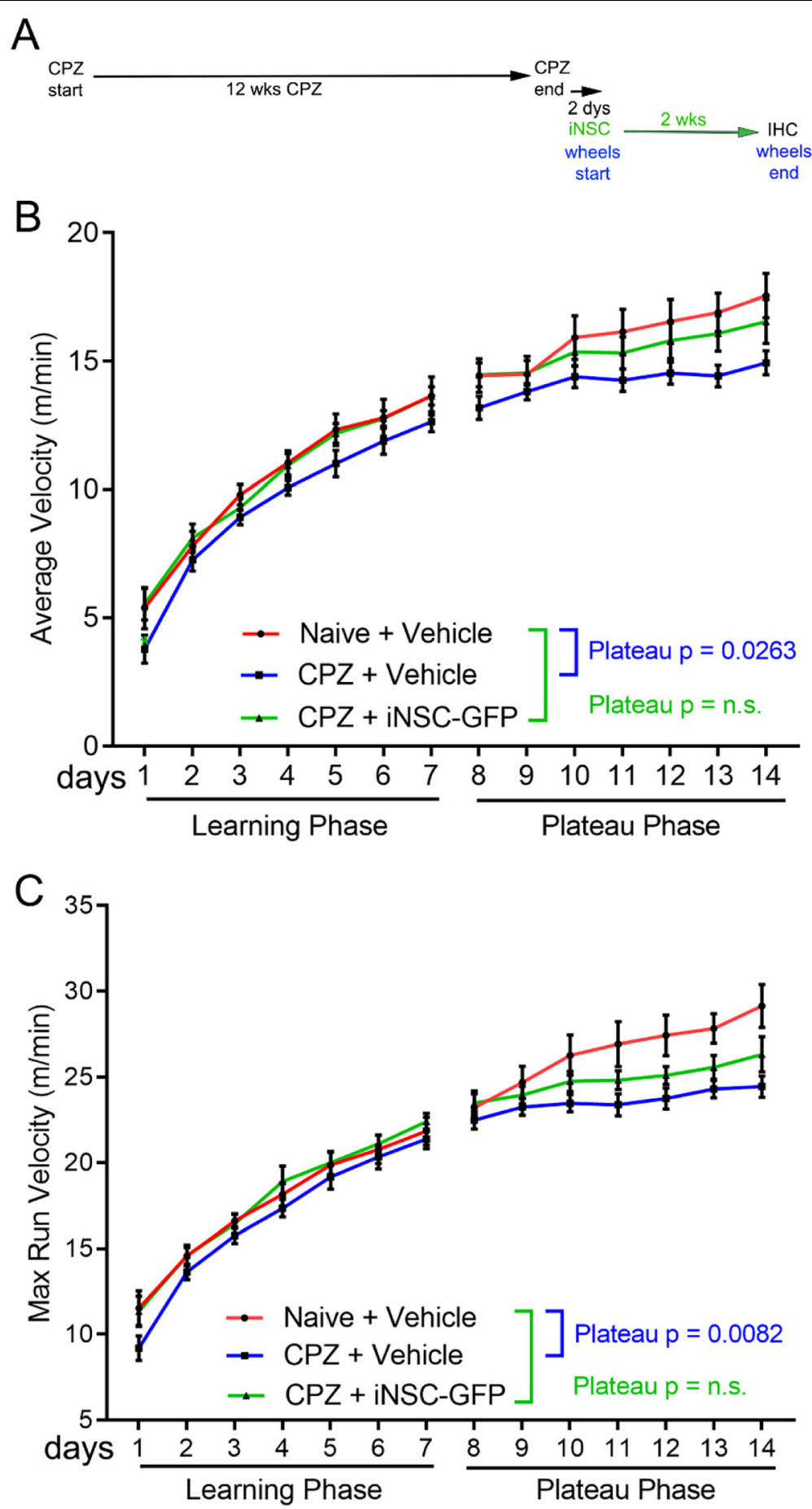

Fig. 7 Recovery of motor function with iNSC transplantation. a: Experimental timeline for functional assessment after iNSC (or vehicle) injection. After 12 weeks of $\mathrm{CPZ}$, mice were returned to normal chow with intracerebral injection performed the following day and exposure to Miss-step wheels the next day. Miss-step wheels with irregularly spaced rungs present a motor skill task mice must learn to run rapidly (week 1); the subsequent plateau velocity reveals deficits of bilateral sensorimotor function (week 2). b-c: The average velocities (b) and the maximum velocities (c) show deficits in vehicle-injected CPZ mice during the learning phase that reach statistical significance during the plateau phase, as compared to naive mice. CPZ mice injected with iNSCs show recovery in that both average and maximum velocities are not significantly different from the naïve mice. Velocity measures were compared using two-way ANOVA with Sidak's multiple comparison test. Data are mean values \pm sem. Data from 3 independent cohorts was combined for analysis of naïve $(n=11)$, CPZ vehicle $(n=12)$, and CPZ iNSC $(n=11)$ 
chronic demyelination, compared to naïve mice (Fig. 7bc), suggesting improved motor learning and performance.

\section{Increased oligodendrocyte response to iNSC transplantation during chronic demyelination}

Following behavior testing, the same mice were analyzed for neuropathology and the oligodendrocyte response to iNSC transplantation (Fig. 8). Overtly, CC atrophy and myelination appeared similar in vehicle injected compared to iNSC transplant mice (Fig. 8a-b). Transplanted iNSCs were observed along the track of the injection, in the $\mathrm{CC}$, cingulum, and adjacent cortex, and rarely in the underlying hippocampus (Fig. 8c). Astrogliosis persisted in the CC after iNSC transplantation and exposure to Miss-step wheels (Fig. 8c). The iNSCs did not express the microglial marker IBA1 and did not markedly alter lipofuscin granules in microglia, which were evident after chronic CPZ with and without iNSC transplantation (Fig. 8d). The oligodendrocyte populations, detected by Plp1 expression, appeared to vary in both density and morphology (Fig. 8e-f). Note that the in situ hybridization technique for Plp1 transcripts is not compatible with immunofluorescence detection of GFP expression to identify iNSCs in these images. Based on quantitative analyses, iNSC transplantation did not attenuate CC atrophy (Fig. 8g), demyelination (Fig. 8h), or astrogliosis (Fig. 8i), compared to vehicle injection. Microglia immunoreactivity was relatively low after the recovery period in both iNSC and vehicle mice (Fig. 8j). Importantly, iNSC transplantation significantly increased the oligodendrocyte cell density compared to vehicle injection (Fig. 8k).

Given the iNSC effect on Plp1, we focused on its expression during the recovery period after chronic CPZ, which appeared to vary in both density and morphology (Fig. 8e, f). Plp1 transcripts were noted mainly in the perinuclear cytoplasm of low expressing oligodendrocytes, but also extended out into processes of oligodendrocytes expressing high levels of Plp1 (Fig. 8f). This observation was supported by several aspects of Plp1 expression indicating potential interest as a dynamic response during remyelination. An increase of Plp1 expressing cells has long been associated with remyelination in MS lesions [65]. Plp1 expression can be regulated by interaction with axons during development [66]. In neonatal rat cultures, Plp1 transcripts are initially localized in oligodendrocyte perinuclear cytoplasm then are also present in primary processes after a delay of several days [67]. In adult mouse white matter, Plp1 transcripts are typically localized in oligodendrocyte cell bodies [68].

Additional analysis explored the effect of iNSC transplantation on the Plp1 expressing oligodendrocyte populations. Oligodendrocytes were distinguished based on
Plp1 expression as $P l p 1^{\text {low }}$ with perinuclear transcript localization, or $P l p 1^{\text {high }}$ with more intense signal in the cell body and extending into processes (Figs. 8e-f and 9). Among the wheel running mice, iNSC transplantion significantly increased $P l p 1^{\text {low }}$ cells, compared to vehicle injected mice (Fig. 9a). After chronic CPZ, OPCs and Ki-67 labeled proliferating cells are relatively rare as compared to the robust proliferation and amplification of OPCs after acute CPZ [20]. However, transplantation of iNSCs increased the frequency of proliferating OPCs during remyelination with wheel access (Fig. 9b-c).

Additional studies were conducted to gain insights as to the $P l p 1^{\text {low }}$ and $P l p 1^{\text {high }}$ populations relative to demyelination and remyelination, additional mice were analyzed without wheel exposure (Fig. 9d-i). In the absence of wheel exposure, Plp ${ }^{\text {low }}$ cells comprised the majority of oligodendrocytes in naïve mice and were significantly reduced after chronic CPZ (Fig. 9d). In contrast, chronic CPZ increased the Plp1 ${ }^{\text {high }}$ population, suggesting an upregulation of Plp1 transcription consistent with early remyelination (9E). Newly formed oligodendrocytes can be identified by high expression of Enpp 6 transcripts during learning in healthy adult mice [57]. The effect of wheel access on Plp1 and Enpp6 oligodendrocyte populations was examined using acute $\mathrm{CPZ}$ demyelination for more extensive remyelination (Fig. 9f-h). Acute CPZ reduced $P l p 1^{\text {low }}$ cells and increased both $P l p 1^{\text {high }}$ and Enpp $6^{\text {high }}$ cells. Wheel access during remyelination simultaneously decreased Enpp $6^{\text {high }}$ cells and increased Plp1 ${ }^{\text {low }}$ cells. These findings can be modeled as stages of maturation during remyelination (Fig. 9i) and suggest that wheel exposure advances maturation to the $P l p 1^{\text {low }}$ phenotype.

Together, these results indicate that iNSC transplantation in combination with motor skill learning on Missstep wheels enhanced the generation of endogenous proliferating OPCs and their differentiation into mature oligodendrocytes.

\section{iNSC differentiation along oligodendrocyte and astrocyte lineages exhibits interaction with host brain after transplantation into chronic lesions}

iNSC location and differentiation were analyzed in mice combined from the MRI and behavior cohorts (Fig. 10). Transplanted iNSCs were located primarily in white matter of the CC or adjacent cingulum (Fig. 10a). A subpopulation of iNSCs maintained expression of Sox2 (Fig. 10b), a neural stem/progenitor cell marker [69, 70]. iNSCs differentiated along the oligodendrocyte lineage, as identified by Olig2 (Fig. 10c), and the astrocyte lineage, as identified by GFAP (Fig. 10d). In the white matter, iNSC differentiation was not significantly different between MRI and wheel cohorts for Olig2 (MRI 24.4\%; wheels 24.4\%; $p=0.8547$ ) or for GFAP (MRI $21.2 \%$; wheels $15.6 \% ; p=0.1673$ ). iNSCs were also found 

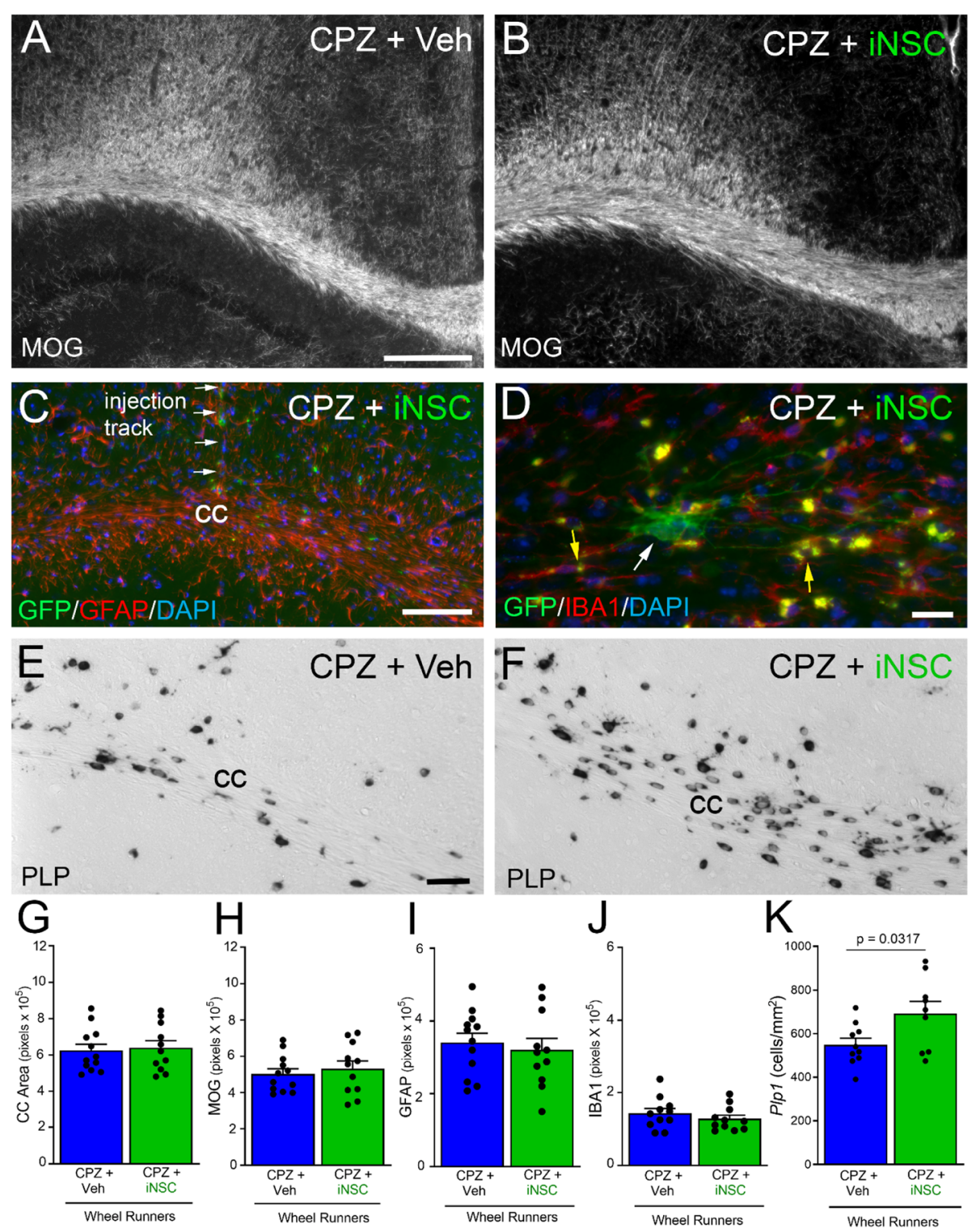

Fig. 8 Post-behavior analysis of CC remyelination and endogenous glial cell response. a-b: Coronal CC sections immunolabeled for MOG to detect myelin in the wheel cohort mice after chronic CPZ with vehicle (a) or iNSC (b) injections. c-d: Innate immune cell response examined with immunolabeling for GFAP (c) or (d). Panel C shows astrogliosis and transplanted iNSCs (green, GFP) within the CC and adjacent areas in a section containing the region of the injection needle track. Panel D shows higher magnification of an iNSC (green, GFP; white arrow) in the CC with thin processes. IBA1 immunolabeled cells contain lipofuscin granules (yellow autofluorescence; yellow arrows). iNSCs did not immunolabel for IBA1. ef: Coronal CC sections processed for in situ hybridization to detect oligodendrocytes expressing proteolipid protein (Plp1). Plp1 appears upregulated after iNSCs transplantation (f) compared to vehicle (e). Plp1 transcripts are localized in the cell body in most cells while a subset of cells show more intense labeling in the cell body and extending into processes. g-k: Quantification of tissue analysis in this post-behavior cohort. CC atrophy and demyelination are not attenuated by iNSCs $(\mathbf{g}, \mathbf{h})$. Transplanted iNSCs did not reduce astrogliosis (i) or the microglial response (j). In contrast, the iNSCS increased the oligodendrocyte population $(\mathbf{k})$. Bar color reflects condition as chronic cuprizone followed by vehicle injection and wheels (blue) or iNSC transplant and wheels (green). Data are mean values \pm sem. Statistical analysis used $t$-tests to compare vehicle and iNSC conditions. For immunolabeling (MOG, GFAP, IBA1), vehicle $n=12$ and iNSC $n=11$. For Plp1 in situ hybridization, vehicle $n=10$, iNSC $n=9$. Scale bars: $A, B$, shown in $A=200 \mu m ; C=100 \mu m ; D=20 \mu m ; E$, $F$, shown in $E=50 \mu m$

in gray matter regions near the needle injection track (Fig. 10a-d). The tissue environment had a marked effect on the iNSC astroglial and oligodendroglial fate of transplanted iNSCs (Fig. 10c-d). iNSC differentiation into oligodendrocyte lineage cells was $31.1 \%$ O4 cells in vitro (Fig. 1d) and 25.6\% Olig2 in white matter but increased significantly to $42.4 \%$ in gray matter (Fig. 10c). iNSC differentiation into astrocytes was $57.3 \%$ under in vitro 


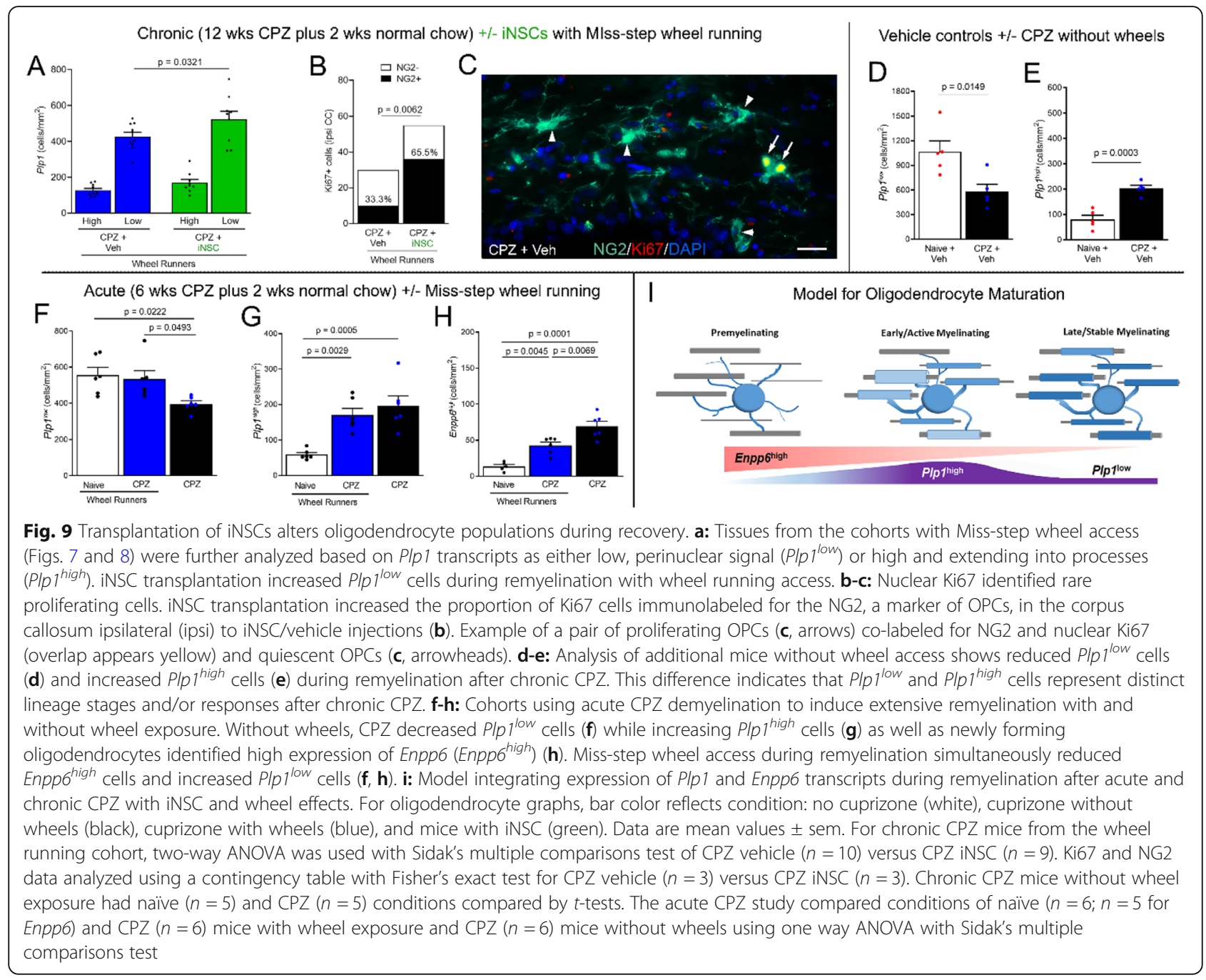

conditions (Fig. 1g). Surprisingly, astrogliogenesis from transplanted iNSCs was reduced to $36.8 \%$ in gray matter and significantly decreased to $17.5 \%$ in white matter after chronic CPZ (Fig. 10d). Immunolabeling for Ki67 detected iNSC proliferation only in a neurosphere formed within the lateral ventricle under the $\mathrm{CC}$ in one mouse (data not shown).

The membrane-targeted GFP revealed iNSC process extension, indicating integration within the host tissue (Fig. 10e-g). Process bearing iNSCs were co-labeled for GFP along with Sox2 (Fig. 10e), Olig2 (Fig. 10f), or GFAP (Fig. 10g) cell type markers, but were not immunolabeled for IBA1 (data not shown). iNSCs were found expressing the neural stem/progenitor marker Nestin, which was not used for quantification due to immunolabeling of extensive processes of reactive astrocytes after chronic CPZ (data not shown), consistent with expression in astrocytes in reactive conditions [71]. Olig2 colabeled iNSCs in the CC extended GFP-labeled membranes parallel to axons but did not clearly form myelin internodes (Fig. 10f), in contrast to our prior studies illustrating GFP labeling of newly formed myelin in NG2CreERT;mTmG mice [72]. In gray matter, GFPlabeled membranes appeared to form astroglial end feet along blood vessels (Fig. 10g).

\section{Axon damage after iNSC transplantation during chronic demyelination}

Mice from wheel running studies were further analyzed to determine whether iNSC transplantation altered the extent of axon damage after chronic CPZ (Fig. 11). Axons were quantified in the cingulum which was demyelinated after chronic CPZ (Fig. 3a-b) and contained transplanted iNSCs (Fig. 10a). As compared to the $\mathrm{CC}$, cingulum axons have the advantages of being distinct populations in each hemisphere that can be quantified in transverse orientation which facilitates quantification in the coronal tissue sections. Neurofilament immunostaining of the overall axon populations showed a slight reduction in the CPZ mice which was 

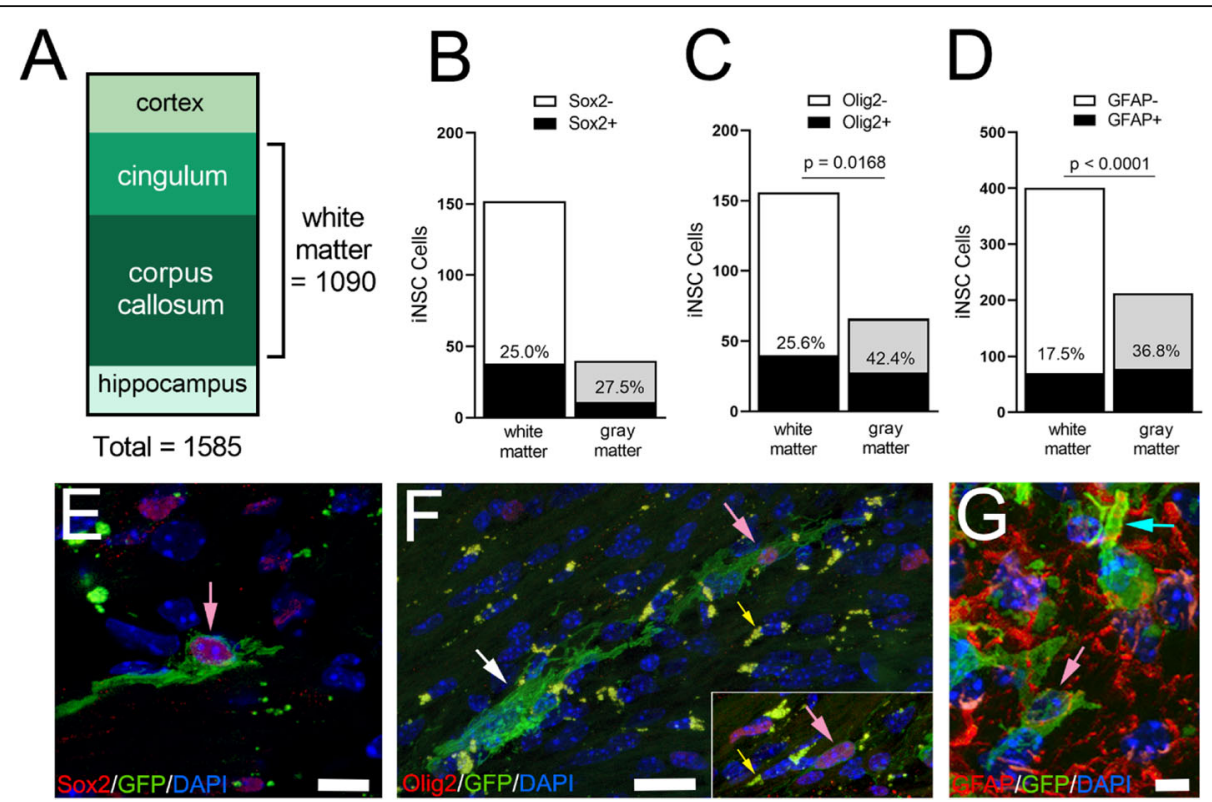

Fig. 10 Transplanted iNSCS are localized mainly in white matter and express markers of oligodendrocyte and astrocyte differentiation. a: The relative distribution of iNSCs in neuroanatomical regions adjacent to the CC target site. iNSCs quantified by direct detection of GFP expression in tissue sections combined from analysis of mice in the MRI and behavior cohorts for neuropathology and iNSC cell type identification. b-d: Quantification of differentiation of transplanted iNSCs in white matter based on direct visualization of GFP expression and co-labeling with markers for neural stem/progenitor cells (Sox2), oligodendrocyte lineage cells (Olig2), or the astrocyte lineage (GFAP). Black fill shows counts of iNSC co-labeled for given cell marker, with upper bar section showing unlabeled iNSC counts. Percent co-labeling shown for each region. e-g: Examples of transplanted iNSCs expressing green fluorescent protein (GFP) along with nuclear Sox2 (e), nuclear Olig2 (f), or cytoplasmic GFAP (g). iNSC membranes labeled for GFP extend around cell bodies (pink arrows), along axons (white arrow), and to blood vessels (blue arrow). iNSCs were not identified as microglia in any sections analyzed with IBA1 immunolabeling. Microglia often contained autofluorescent lipofuscin granules (f, yellow arrows). Tissue sections from mice of both the MRI $(n=6)$ and behavior $(n=11)$ cohorts were combined for the analysis of iNSC distribution. Differentiation studies included a subset of mice (Sox2 $(n=6)$, Olig2 $(n=9)$, and GFAP $(n=14))$ combined from MRI and behavior cohorts to generate contingency tables of total cell counts among sections analyzed for comparison using Fisher's exact test. Scale bars $\mathrm{E}=10 \mu \mathrm{m} ; \mathrm{F}=20 \mu \mathrm{m} ; \mathrm{G}=5 \mu \mathrm{m}$

not significant (Fig. 11a). Co-immunolabeling of nonphosphorylated neurofilaments with SMI32 identifies damaged axons after chronic CPZ [41]. Naïve and CPZ mice with vehicle injections show that the injection procedure did not alter axons on the ipsilateral side (Fig. 11a-b). The proportion of damaged axons is significantly increased after $\mathrm{CPZ}$ in mice injected with vehicle, but not in mice with iNSC transplants (Fig. 11b). Nuclear labeling with DAPI, as an estimate of changes in cellularity, was increased after CPZ in mice receiving vehicle or iNSC transplantation (Fig. 11c). Similar to findings for the CC (Fig. 9b-c), rare proliferating cells identified by Ki67 showed a significant increase in OPCs immunolabeled for NG2 (Fig. 11d). Together, these findings indicate that exogenous iNSCs modulate multiple processes during remyelination.

\section{Discussion}

This study evaluated the potential for transplantation of iNSCs to enhance recovery at a chronic stage of demyelination. MRI and neurological outcome measures clearly demonstrated structural and functional deficits from CPZ-mediated demyelination. MRI cohorts with iNSCs showed reduced DTI RD, a parameter often associated with aspects of remyelination after $\mathrm{CPZ}$ demyelination $[61,73]$. Transplantation of iNSCs improved running velocities during a Miss-step wheel task, which is relevant to CC function [35].

Our study is the first to show improved performance on a neurologic outcome measure following transplantation of brain stem cells into the highly astrogliotic demyelinated lesions produced by chronic CPZ.

\section{Chronic CPZ model}

The CPZ model is advantageous for testing cell-based therapeutics after chronic demyelination. Oligodendrocyte loss from CPZ toxicity facilitates testing cell replacement from transplanted cells. CPZ pathology is similar to MS lesions, specifically pattern III pathology with oligodendrogliopathy and low lymphocyte infiltration [18, 74-76]. CPZ produces extensive astrogliosis that persists through chronic demyelination [23]. In addition to demyelination, CPZ results in axon damage that forms varicosities from disrupted rapid axonal 


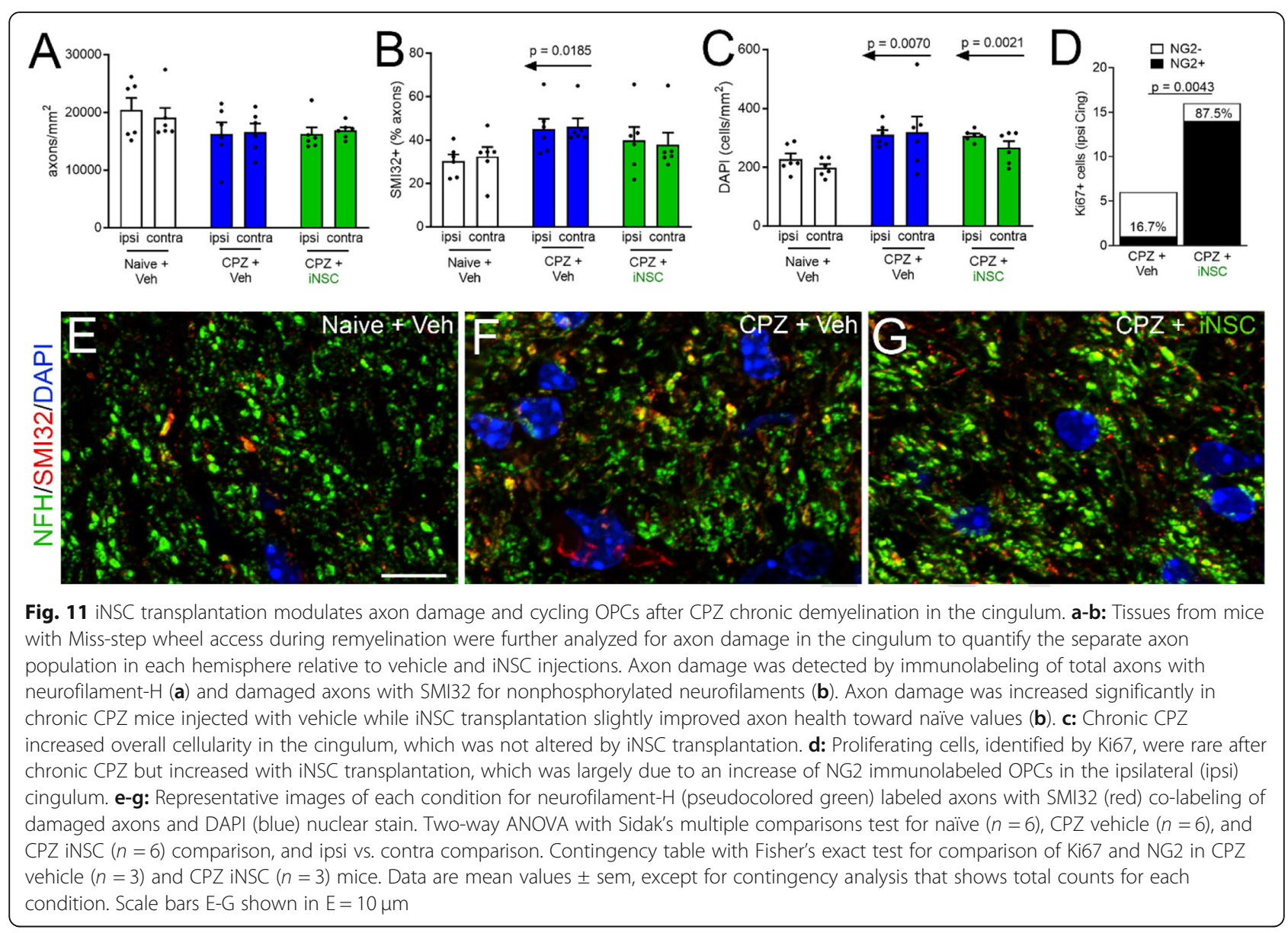

transport during the acute stage that progresses to neurofilament dephosporylation and reduced axon diameters after chronic CPZ [40, 41]. In patients with MS, CC atrophy correlates with functional outcomes [25, 26]. Chronic CPZ exhibits CC atrophy, without decreasing neurons in the cerebral cortex [24]. However, in the current experiments, transplantation of iNSCs improved function along with a moderate benefit on the oligodendrocyte lineage response and axon damage, while not reducing overall CC demyelination or atrophy.

\section{Neuroimaging}

MRI distinguishes $\mathrm{CPZ}$ disease stages with predominant axon damage, demyelination, or remyelination $[40,77$, 78]. DTI and other measures of CC integrity correlate with functional measures in MS patients [79]. Decreased DTI AD during acute CPZ (4 weeks) corresponds with axonal varicosities and increased cellularity, but is less effected with longer CPZ administration [40, 63]. DTI $\mathrm{RD}$ increased during $\mathrm{CPZ}$ demyelination, and decreased with improved remyelination in mice with genetic deletion of $F g f 2$ or with estrogen receptor $\beta$ ligand treatment $[41,63]$. The current longitudinal studies complemented DTI with quantitative T2 and MTR. Our results agree with reports of increased DTI RD and T2 values along with decreased MTR during CPZ demyelination [40, 73]. Furthermore, mice with transplanted iNSCs had reduced DTI RD during remyelination. Although the only MRI parameter exhibiting a significant change from iNSC transplant, this difference in DTI RD has robust statistical power (96.3\%) and effect size (Cohen's $d=3.74$ ). Post-imaging pathology did not detect a difference in overall CC remyelination within this MRI cohort. DTI can be influenced by cellularity or edema [64]. Therefore, iNSC effects on DTI RD may involve the reduced astrogliosis, which can contribute to local anisotropy. However, subtle changes to axon-myelin structure could also be involved yet not detected by our post-imaging analysis techniques. For example, T2 and DTI revealed abnormalities in CST-KO mice that have detached myelin at paranodes, without overt demyelination [80].

\section{Functional outcome measure}

After chronic CPZ iNSC transplantation promoted recovery as detected using the Miss-step wheel running task. Miss-step wheels have a non-uniform pattern of missing rungs. Mice learn to avoid stepping faults by placing a hind paw onto a rung previously grasped by a 
forepaw; learning this motor skill stimulates myelination from CC oligodendrocyte lineage cells in healthy adult mice [35]. The population of newly forming oligodendrocytes, expressing Enpp6, indicated interactive effects of Miss-step wheels on endogenous cells during remyelination. Learning this skilled motor task may have contributed to effects on oligodendrocyte differentiation, since neuronal activity can modulate both new and existing oligodendrocytes to contribute to remyelination $[81,82]$. After the learning phase, deficits in plateau running velocity correspond with myelination status [33, 35]. This Miss-step wheel running may also work as a targeted exercise enrichment, acting in conjunction with iNSC transplantation to promote recovery. For example, normal running wheels acted in parallel with clemastine, a pro-remyelinating treatment, to additively enhance remyelination after lysolecithin demyelination [15].

Together, these findings suggest an intrinsic regenerative potential for iNSC-based therapeutics and anticipate further studies to examine the interaction between targeted enrichment strategies and advanced regenerative medicines that are targeted to improve function.

\section{Host endogenous cell responses}

The cohorts that exhibited improved function after iNSC transplantation also had increased populations of host endogenous oligodendrocytes. Transplantation of iNSCs increased the total Plp1 expressing cells in the CC among the mice that showed improvement of Miss-step wheel running. The iNSC transplants significantly increased the Plp1 low population and may have transitioned from the Plp $1^{\text {high }}$ population. The current results are the first to show that both $P l p 1^{\text {high }}$ and Enpp $6^{\text {high }}$ cells increase during remyelination. Mice with iNSC transplantation and wheel access also had an increased proliferative response that was mainly comprised of OPCs. Thus, iNSCs may act in conjunction with Missstep wheel running to promote differentiation of endogenous OPCs into oligodendrocytes. This interpretation is also in agreement with the slight increase of Plp1 expressing cells after iNSC transplantation in the MRI cohort.

Chronic CPZ produced persistent CC neuroinflammation, with astrogliosis being more marked than microglial activation. Astrogliosis may mediate beneficial and detrimental effects on remyelination and axon degeneration in the progression to chronic demyelination in $\mathrm{CPZ}$ and in MS [23, 83, 84]. Astrogliosis was significantly reduced by iNSC transplantation in the MRI cohort. However, this GFAP reduction was not found in the wheel cohort. Therefore, interpreting effects of iNSC transplantation and/or wheel exposure may require deeper analysis of distinct astrocyte phenotypes, which have been reported to switch during early vs. late remyelination in an autoimmune model of demyelination in rats [85].

One potential explanation for these differences in Plp1 and GFAP analyses in the MRI and wheel cohorts could be that wheel running may elicit an interaction between the iNSC transplants and the host cells or tissue environment. An alternative to consider is the potential for false positive results when the statistical power drops below the conventional $80 \%$ level [86]. Due to the death of one mouse, the power for the GFAP analysis in the MRI iNSC cohort fell to $74.4 \%$, yet with an effect size of 1.62 (Cohen's $d$ ). The increase of Plp1 cells after iNSC transplantation in the wheel cohort was $62.3 \%$, with an effect size of 1.09 .

\section{Stem cell-based therapeutics}

The current approach of direct conversion of mouse somatic cells, e.g. fibroblasts, into iNSCs provides stably expandable populations, and is feasible for generating human iNSCs [29-32]. Direct conversion of fibroblasts circumvents the pluripotent state, which delivers autologous NSCs more rapidly than by iPSC-type reprogramming and subsequent differentiation and is known to reduce neoplastic potential and increase genomic stability [87]. Human iNSC can be either derived by reprogramming from adult dermal fibroblasts [30] or peripheral blood monocytes [32]. Preclinical research has revealed an unexpected ability of iNSC therapies to provide neurotrophic support and inhibit detrimental host immune responses in vivo, after transplantation into the chronically inflamed CNS [88].

After transplantation into chronic CPZ lesions, iNSCs remained undifferentiated, or differentiated along oligodendrocyte or astrocyte lineages; the tissue environment significantly modulated glial differentiation (Fig. 10). iNSCs did not appear to form substantial new myelin within 2 weeks post-transplantation. Thus, iNSCs likely improved function by stimulating endogenous cell responses. As with NSCs generated by other means, iNSCs may exhibit beneficial paracrine effects on endogenous cells [89].

A recent review of cell-based therapeutic strategies for MS indicated that the preferred course of action for clinical translation may be through beneficial paracrine effects from stem cells, rather than direct cell replacement requiring injections into multiple lesion sites $[88,90]$. When applied to NSC therapeutics, most preclinical studies have found cell replacement to be secondary to other "bystander" effects, in which transplanted NSCs modulate homeostasis favoring neuroprotection and immunomodulation via multiple mechanisms. NSCs in fact exert direct neuroprotective action through the secretion of neurotrophic factors; NSCs also inhibit the peripheral and perivascular activation of pro-inflammatory $\mathrm{T}$ cells, while 
increasing numbers of inflammatory $\mathrm{T}$ regulatory cells. In the context of progressive MS, mechanisms of action of NSCs on mononuclear phagocytes are emerging to be of pivotal importance. Among these, the reprogramming of pro-inflammatory mononuclear phagocytes through sequestration of the immunomodulatory metabolite succinate and secretion of PGE2 is common to both iNSCs and NSCs [51].

Several studies have transplanted cells into the lateral ventricle in the chronic CPZ model and reported beneficial effects without direct cell replacement. Transplanting neural precursor cells into the ventricle after chronic CPZ stimulated the endogenous OP response and improved CC remyelination [91]. Mesenchymal stem cells transplanted after chronic CPZ exhibited immunosuppressive effects on cytokines, astrocytes and microglia; this approach also activated subventricular zone NSCs and increased CC oligodendrocyte lineage cells, remyelination, and ex vivo measures of conduction velocity [92, 93]. Furthermore, NSC-Lingo-1-Fc cells, which antagonize Lingo1 , injected via tail vein during chronic experimental autoimmune encephalomyelitis effectively alleviated neurologic symptoms [94]. These findings indicate beneficial effects of stem cell transplants during chronic disease across MS models. Recently, extensive remyelination in the CPZ model was achieved with transplantation of human glial progenitor cells [95]. The human cells were transplanted during periods of OPC amplification in postnatal mice or at 4 weeks of $\mathrm{CPZ}$ ingestion, and so do not address transplantation into a chronic host lesion environment. Remarkably, the transplanted cells generated widespread myelin after a further 16 weeks of CPZ ingestion.

\section{Study limitations}

Certain limitations of the current study are important to interpretation of the data. The chronic CPZ model provides a highly astrogliotic demyelinated lesion yet cannot fully replicate the pathological mechanisms of demyelination and neurodegeneration of progressive MS [76]. In addition, MS lesions are distributed within both white matter and gray matter regions [76]. Some iNSCs were localized in cerebral cortex or hippocampus, which are regions that can exhibit demyelination from CPZ [33, 96, 97], but our techniques were not designed for analysis of gray matter sites. In MS populations, sex is an important biological variable that cannot be appropriately examined in the CPZ model in C57BL/6 mice since CPZ disrupts the estrus cycle [36]. A 2-week period after transplantation was used to capture early iNSC responses in this chronic lesion environment while a longer interval could more fully examine iNSC remyelinating potential. Furthermore, comparison between mouse and human iNSC direct and/ or paracrine effects will be important in future studies.

\section{Conclusions}

These results show that iNSC transplantation can enhance recovery of function even in chronically demyelinated white matter that already exhibits significant atrophy. Our results suggest a combination of subtle beneficial effects from interactions between iNSCs and the lesion environment that promote functional recovery. As cell replacement was not required, similar effects may be achievable using small molecules and/or iNSC exosomes [89].

Altogether, despite compelling preclinical evidence of the therapeutic effects of transplantation in animal models of demyelinating diseases, further research is warranted to evaluate mechanism(s) of action from iNSC transplantation, and how to exploit these mechanisms for translation to treatments for progressive MS.

\section{Acknowledgements}

The authors thank Tuan Q. Le, Xiaomei Zi, Dr. Fengshan Yu, and Dr. Christina Marion for technical assistance. We appreciate the support of the Center for Neuroscience and Regenerative Medicine Translational Imaging Core and the Biomedical Instrumentation Center at the Uniformed Services University. These studies were funded by the U.S. Congressionally Directed Medical Research Program (W81XWH-15-2-0081; MS140019 S.P, MS140019P1 R.C.A, MS14009P2 F.E.). LPJ was supported by a Wellcome Trust Research Training Fellowship [RRZA/057 RG79423] and a senior research fellowship FISM Fondazione Italiana Sclerosi Multipla - cod. 2017/B/5 financed or co-financed with the '5 per mille' public funding and Addenbrooke's Charitable Trust (RG 97519).

\section{Data sets}

The techniques used did not generate data sets such as those expected in available repositories.

\section{Authors' contributions}

GMS, RCA, LPJ, and SP designed studies of the cell transplantation into the chronic cuprizone model. RCA, GMS, LPJ. and SP designed transplanted cell type analysis of tissue sections. RCA and GMS designed neuropathological analysis of tissue sections. GMS performed cuprizone experiments and neuropathological analyses. GMS characterized expanded iNSCS according to protocols established by LPJ, JDB, SP and FE GMS performed intracerebral transplant experiments. FE designed procedures and generated initial mouse induced neural stem cell preparations and provided protocols for cell expansion and characterization. SP designed procedures and provided protocols for in vitro cell expansion and characterization. LPJ and JDB characterized and expanded iNSCs in vitro; generated lentiviral vectors for iNSC transduction; transduced, expanded and characterized iNSCs expressing farnesylated GFP in vitro. RCA, GMS, AKK, AK, AB, and BJD designed MRI studies for cuprizone and transplants. GMS, AK, AB performed MRI studies. GMS, RCA, AKK, AK, and BJD analyzed MRI data. GMS performed behavioral studies. GMS and RCA designed and analyzed behavioral studies. RCA conceived of the experiments and secured funding for project, in collaboration with FE and SP, under a program project led by SP GMS and RCA wrote the manuscript. All authors edited and approved the manuscript.

\section{Competing interests}

S.P. is co-founder and CSO at CITC Ltd. and iSTEM Therapeutics, and COfounder and Non-executive Director at Asitia Therapeutics. L.P.J. is Head of Research at iSTEM Therapeutics.

\section{Author details}

'Department of Anatomy, Physiology and Genetics, F. Edward Hebert School of Medicine, Uniformed Services University of the Health Sciences, 4301 Jones Bridge Rd, Bethesda, MD 20814, USA. ${ }^{2}$ Center for Neuroscience and Regenerative Medicine, F. Edward Hebert School of Medicine, Uniformed Services University of the Health Sciences, Bethesda, MD 20814, USA. 
${ }^{3}$ Department of Radiology and Radiological Sciences, F. Edward Hebert School of Medicine, Uniformed Services University of the Health Sciences, Bethesda, MD 20814, USA. ${ }^{4}$ Department of Clinical Neurosciences and NIHR Biomedical Research Centre, University of Cambridge, Cambridge, UK. ${ }^{5}$ Present address: Department of Neurosurgery, Brigham and Women's Hospital, Harvard Medical School, Boston, MA, USA. ${ }^{6}$ Genomics, Stem Cell Biology and Regenerative Medicine \& CMBI, Leopold-Franzens-University Innsbruck, Innsbruck, Austria.

Received: 28 May 2020 Accepted: 30 May 2020

\section{Published online: 09 June 2020}

\section{References}

1. Wallin MT et al (2019) The prevalence of MS in the United States: a population-based estimate using health claims data. Neurology 92(10): e1029-e1040

2. Plemel JR, Liu WQ, Yong WW (2017) Remyelination therapies: a new direction and challenge in multiple sclerosis. Nat Rev Drug Discov 16(9): 617-634

3. Plemel JR et al (2014) Remyelination after spinal cord injury: is it a target for repair? Prog Neurobiol 117:54-72

4. Bove RM, Green AJ (2017) Remyelinating pharmacotherapies in multiple sclerosis. Neurotherapeutics 14(4):894-904

5. Abu-Rub M, Miller RH (2018) Emerging cellular and molecular strategies for enhancing central nervous system (CNS) remyelination. Brain Sci 8(6):111

6. Faissner $\mathrm{S}$ et al (2019) Progressive multiple sclerosis: from pathophysiology to therapeutic strategies. Nat Rev Drug Discov 18:905-922

7. Armstrong RC et al (1992) Pre-oligodendrocytes from adult human CNS. J Neurosci 12(4):1538-1547

8. Chang A et al (2002) Premyelinating oligodendrocytes in chronic lesions of multiple sclerosis. N Engl J Med 346(3):165-173

9. Kuhlmann T et al (2008) Differentiation block of oligodendroglial progenitor cells as a cause for remyelination failure in chronic multiple sclerosis. Brain 131(Pt 7):1749-1758

10. Green AJ et al (2017) Clemastine fumarate as a remyelinating therapy for multiple sclerosis (ReBUILD): a randomised, controlled, double-blind, crossover trial. Lancet 390(10111):2481-2489

11. Cadavid D et al (2019) Safety and efficacy of opicinumab in patients with relapsing multiple sclerosis (SYNERGY): a randomised, placebo-controlled, phase 2 trial. Lancet Neurol 18(9):845-856

12. Mi $\mathrm{S}$ et al (2009) Promotion of central nervous system remyelination by induced differentiation of oligodendrocyte precursor cells. Ann Neurol 65(3): 304-315

13. Mei F et al (2014) Micropillar arrays as a high-throughput screening platform for therapeutics in multiple sclerosis. Nat Med 20(8):954-960

14. Shao Z et al (2017) LINGO-1 regulates Oligodendrocyte differentiation through the cytoplasmic Gelsolin signaling pathway. J Neurosci 37(12): 3127-3137

15. Jensen SK et al (2018) Multimodal enhancement of Remyelination by exercise with a pivotal role for Oligodendroglial PGC1alpha. Cell Rep 24(12): 3167-3179

16. Lassmann H, Bradl M (2017) Multiple sclerosis: experimental models and reality. Acta Neuropathol 133(2):223-244

17. Mason JL et al (2001) Episodic demyelination and subsequent remyelination within the murine central nervous system: changes in axonal calibre. Neuropathol Appl Neurobiol 27(1):50-58

18. Kipp $\mathrm{M}$ et al (2009) The cuprizone animal model: new insights into an old story. Acta Neuropathol 118(6):723-736

19. Mason JL et al (2000) Mature oligodendrocyte apoptosis precedes IGF-1 production and oligodendrocyte progenitor accumulation and differentiation during demyelination/remyelination. J Neurosci Res 61(3): $251-262$

20. Armstrong RC et al (2006) Endogenous cell repair of chronic demyelination. J Neuropathol Exp Neurol 65(3):245-256

21. Huang Y, Dreyfus CF (2016) The role of growth factors as a therapeutic approach to demyelinating disease. Exp Neurol 283(Pt B):531-540

22. Mason $\mathrm{J}$ et al (2004) Oligodendrocytes and progenitors become progressively depleted within chronically demyelinated lesions. Am J Pathol 164(5):1673-1682

23. Hibbits $\mathrm{N}$ et al (2012) Astrogliosis during acute and chronic cuprizone demyelination and implications for remyelination. ASN Neuro 4(6):393-408
24. Hochstrasser T et al (2019) Stereological investigation of regional brain volumes after acute and chronic cuprizone-induced demyelination. Cells 8(9):1024

25. Yaldizli $O$ et al (2014) The relationship between total and regional corpus callosum atrophy, cognitive impairment and fatigue in multiple sclerosis patients. Mult Scler 20(3):356-364

26. Granberg $T$ et al (2015) Corpus callosum atrophy is strongly associated with cognitive impairment in multiple sclerosis: results of a 17-year longitudinal study. Mult Scler 21(9):1151-1158

27. Zhou YX et al (2012) Fibroblast growth factor 1 (FGFR1) modulation regulates repair capacity of oligodendrocyte progenitor cells following chronic demyelination. Neurobiol Dis 45(1):196-205

28. Gudi V et al (2014) Glial response during cuprizone-induced de- and remyelination in the CNS: lessons learned. Front Cell Neurosci 8:73

29. Thier $\mathrm{M}$ et al (2012) Direct conversion of fibroblasts into stably expandable neural stem cells. Cell Stem Cell 10(4):473-479

30. Meyer $\mathrm{S}$ et al (2015) Derivation of adult human fibroblasts and their direct conversion into expandable neural progenitor cells. J Vis Exp 101:e52831

31. Erharter A et al (2019) Take the shortcut: direct conversion of somatic cells into induced neural stem cells and their biomedical applications. FEBS Lett 593:3353-3369

32. Thier MC et al (2019) Identification of embryonic neural plate border stem cells and their generation by direct reprogramming from adult human blood cells. Cell Stem Cell 24(1):166-182 e13

33. Hibbits $\mathrm{N}$ et al (2009) Cuprizone demyelination of the corpus callosum in mice correlates with altered social interaction and impaired bilateral sensorimotor coordination. ASN Neuro 1(3):AN20090032

34. Mierzwa AJ et al (2013) FGF2 and FGFR1 signaling regulate functional recovery following cuprizone demyelination. Neurosci Lett 548:280-285

35. McKenzie IA et al (2014) Motor skill learning requires active central myelination. Science 346(6207):318-322

36. Taylor LC et al (2010) Cuprizone induces similar demyelination in male and female C57BL/6 mice and results in disruption of the estrous cycle. J Neurosci Res 88(2):391-402

37. Hochstrasser $\mathrm{T}$ et al (2017) Cuprizone-containing pellets are less potent to induce consistent demyelination in the Corpus callosum of C57BL/6 mice. J Mol Neurosci 61(4):617-624

38. Davidson NL et al (2017) Leukemia/lymphoma-related factor (LRF) exhibits stage- and context-dependent transcriptional controls in the oligodendrocyte lineage and modulates remyelination. J Neurosci Res 95(12):2391-2408

39. Sanchez MA, Sullivan GM, Armstrong RC (2018) Genetic detection of sonic hedgehog (Shh) expression and cellular response in the progression of acute through chronic demyelination and remyelination. Neurobiol Dis 115: 145-156

40. Xie M et al (2010) Rostrocaudal analysis of corpus callosum demyelination and axon damage across disease stages refines diffusion tensor imaging correlations with pathological features. J Neuropathol Exp Neurol 69(7):704-716

41. Tobin JE et al (2011) Reduced axonopathy and enhanced remyelination after chronic demyelination in fibroblast growth factor 2 (Fgf2)-null mice: differential detection with diffusion tensor imaging. J Neuropathol Exp Neurol 70(2):157-165

42. Yu F et al (2017) Repetitive model of mild traumatic brain injury produces cortical abnormalities detectable by magnetic resonance diffusion imaging (DTI/DKI), histopathology, and behavior. J Neurotrauma 34:1364-1381

43. Basser PJ, Pierpaoli C (1996) Microstructural and physiological features of tissues elucidated by quantitative-diffusion-tensor MRI. J Magn Reson B 111(3):209-219

44. Hennig J, Nauerth A, Friedburg H (1986) RARE imaging: a fast imaging method for clinical MR. Magn Reson Med 3(6):823-833

45. Dousset $V$ et al (1992) Experimental allergic encephalomyelitis and multiple sclerosis: lesion characterization with magnetization transfer imaging. Radiology 182(2):483-491

46. Tu TW et al (2016) Radiological-pathological correlation of diffusion tensor and magnetization transfer imaging in a closed head traumatic brain injury model. Ann Neurol 79(6):907-920

47. Sullivan GM et al (2013) Oligodendrocyte lineage and subventricular zone response to traumatic axonal injury in the corpus callosum. J Neuropathol Exp Neurol 72(12):1106-1125

48. Pierpaoli $C$ et al. (2010) TORTOISE: an integrated software package for processing of diffusion MRI data. ISMRM. 18th annual meeting: p. 1597 
49. Irfanoglu MO et al. (2017) TORTOISEv3: Improvements and new features of the NIH diffusion MRI processing pipeline. ISMRM. 25th Annual Meeting: $p$. 3540

50. Chang LC, Jones DK, Pierpaoli C (2005) RESTORE: robust estimation of tensors by outlier rejection. Magn Reson Med 53(5):1088-1095

51. Peruzzotti-Jametti $L$ et al (2018) Macrophage-derived extracellular succinate licenses neural stem cells to suppress chronic neuroinflammation. Cell Stem Cell 22(3):355-368 e13

52. Armstrong, RC (1998) Isolation and characterization of immature oligodendrocyte lineage cells. In: Techniques for Purification, Functional Evaluation and Transplantation of Brain Cells, R. Rozental and F-C Chiu (eds), Academic Press, NY. Methods: A Companion to Methods in Enzymology 16(3):282-292

53. Matsubayashi $Y$ et al (2009) Immunostaining for oligodendrocyte-specific galactosphingolipids in fixed brain sections using the cholesterol-selective detergent digitonin. J Neurosci Methods 178(1):87-98

54. Sullivan GM, Armstrong RC (2017) Transplanted adult neural stem cells express sonic hedgehog in vivo and suppress white matter neuroinflammation after experimental traumatic brain injury. Stem Cells Int 2017:9342534

55. Armstrong RC et al (2002) Absence of fibroblast growth factor 2 promotes oligodendroglial repopulation of demyelinated white matter. J Neurosci 22(19):8574-8585

56. Redwine JM, Armstrong RC (1998) In vivo proliferation of oligodendrocyte progenitors expressing PDGFalphaR during early remyelination. J Neurobiol 37(3):413-428

57. Xiao L et al (2016) Rapid production of new oligodendrocytes is required in the earliest stages of motor-skill learning. Nat Neurosci 19(9):1210-1217

58. Budde MD, Frank JA (2012) Examining brain microstructure using structure tensor analysis of histological sections. Neuroimage 63(1):1-10

59. Szczepankiewicz F et al (2015) Quantification of microscopic diffusion anisotropy disentangles effects of orientation dispersion from microstructure: applications in healthy volunteers and in brain tumors. Neuroimage 104:241-252

60. Armstrong RC, Redwine JM, Messersmith DJ (2005) Coronavirus-induced demyelination and spontaneous remyelination: growth factor expression and function. In: Lavi E, Constantinescu CS (eds) Experimental models of multiple sclerosis. Kluver, Norwell In press

61. Song SK et al (2005) Demyelination increases radial diffusivity in corpus callosum of mouse brain. Neuroimage 26(1):132-140

62. Sun SW et al (2006) Noninvasive detection of cuprizone induced axonal damage and demyelination in the mouse corpus callosum. Magn Reson Med 55(2):302-308

63. Atkinson KC et al (2019) Diffusion tensor imaging identifies aspects of therapeutic estrogen receptor beta ligand-induced remyelination in a mouse model of multiple sclerosis. Neurobiol Dis 130:104501

64. Wang $Y$ et al (2011) Quantification of increased cellularity during inflammatory demyelination. Brain 134(Pt 12):3590-3601

65. Mews I et al (1998) Oligodendrocyte and axon pathology in clinically silent multiple sclerosis lesions. Mult Scler 4(2):55-62

66. Thomson CE et al (2005) Plp gene regulation in the developing murine optic nerve: correlation with oligodendroglial process alignment along the axons. Dev Neurosci 27(1):27-36

67. de Vries $\mathrm{H}$ et al (1997) Differential and cell development-dependent localization of myelin mRNAs in oligodendrocytes. J Neurosci Res 47(5):479-488

68. Jordan C, Friedrich V Jr, Dubois-Dalcq M (1989) In situ hybridization analysis of myelin gene transcripts in developing mouse spinal cord. J Neurosci 9(1): 248-257

69. Cui CP et al (2019) Author correction: dynamic ubiquitylation of Sox2 regulates proteostasis and governs neural progenitor cell differentiation. Nat Commun 10(1):173

70. Suh $\mathrm{H}$ et al (2007) In vivo fate analysis reveals the multipotent and selfrenewal capacities of Sox $2+$ neural stem cells in the adult hippocampus Cell Stem Cell 1(5):515-528

71. Gallo V, Armstrong RC (1995) Developmental and growth factor-induced regulation of nestin in oligodendrocyte lineage cells. J Neurosci 15(1 Pt 1): 394-406

72. Marion CM et al (2018) Experimental traumatic brain injury identifies distinct early and late phase axonal conduction deficits of white matter pathophysiology, and reveals intervening recovery. J Neurosci 38(41):87238736
73. Thiessen JD et al (2013) Quantitative MRI and ultrastructural examination of the cuprizone mouse model of demyelination. NMR Biomed 26(11):15621581

74. Ludwin SK, Johnson ES (1981) Evidence for a "dying-back" gliopathy in demyelinating disease. Ann Neurol 9(3):301-305

75. Lucchinetti $C$ et al (2000) Heterogeneity of multiple sclerosis lesions: implications for the pathogenesis of demyelination. Ann Neurol 47(6):707717

76. Reich DS, Lucchinetti CF, Calabresi PA (2018) Multiple sclerosis. N Engl J Med 378(2):169-180

77. Falangola MF et al (2014) Histological correlation of diffusional kurtosis and white matter modeling metrics in cuprizone-induced corpus callosum demyelination. NMR Biomed 27(8):948-957

78. Turati $L$ et al (2015) In vivo quantitative magnetization transfer imaging correlates with histology during de- and remyelination in cuprizone-treated mice. NMR Biomed 28(3):327-337

79. Huang SY et al (2019) Corpus callosum axon diameter relates to cognitive impairment in multiple sclerosis. Ann Clin Transl Neurol 6(5):882-892

80. Takano M et al (2012) MRI characterization of paranodal junction failure and related spinal cord changes in mice. PLoS One 7(12):e52904

81. Ortiz FC et al (2019) Neuronal activity in vivo enhances functional myelin repair. JCl Insight 5(9):e123434 https://doi.org/10.1172/jci.insight.123434

82. Bacmeister CM et al (2020) Motor learning promotes remyelination via new and surviving oligodendrocytes. Nat Neurosci https://doi.org/10.1038/ s41593-020-0637-3

83. Correale J, Farez MF (2015) The role of astrocytes in multiple sclerosis progression. Front Neurol 6:180

84. Sofroniew MV, Vinters HV (2010) Astrocytes: biology and pathology. Acta Neuropathol 119(1):7-35

85. Haindl MT et al (2019) The formation of a glial scar does not prohibit remyelination in an animal model of multiple sclerosis. Glia 67(3):467-481

86. Lakens D (2013) Calculating and reporting effect sizes to facilitate cumulative science: a practical primer for t-tests and ANOVAs. Front Psychol 4:863

87. Gao M et al (2016) Tumourigenicity and immunogenicity of induced neural stem cell grafts versus induced pluripotent stem cell grafts in syngeneic mouse brain. Sci Rep 6:29955

88. Pluchino S, Smith JA, Peruzzotti-Jametti L (2020) Promises and limitations of neural stem cell therapies for progressive multiple sclerosis. Trends Mol https://doi.org/10.1016/j.molmed.2020.04.005

89. Willis CM et al (2020) The neural stem cell secretome and its role in brain repair. Brain Res 1729:146615

90. Scolding NJ et al (2017) Cell-based therapeutic strategies for multiple sclerosis. Brain 140(11):2776-2796

91. Einstein $O$ et al (2009) Transplanted neural precursors enhance host brainderived myelin regeneration. J Neurosci 29(50):15694-15702

92. Cruz-Martinez P et al (2016) Intraventricular injections of mesenchymal stem cells activate endogenous functional remyelination in a chronic demyelinating murine model. Cell Death Dis 7:e2223

93. Barati S et al (2019) Effect of mesenchymal stem cells on glial cells population in cuprizone induced demyelination model. Neuropeptides 75: 75-84

94. Li X et al (2017) LINGO-1-fc-transduced neural stem cells are effective therapy for chronic stage experimental autoimmune encephalomyelitis. Mol Neurobiol 54(6):4365-4378

95. Windrem MS et al (2020) Human glial progenitor cells effectively remyelinate the demyelinated adult brain. Cell Rep 31(7):107658

96. Buschmann JP et al (2012) Inflammatory response and chemokine expression in the white matter corpus callosum and gray matter cortex region during cuprizone-induced demyelination. J Mol Neurosci 48(1):66-76

97. Goldberg J et al (2015) Anatomical distribution of Cuprizone-induced lesions in C57BL6 mice. J Mol Neurosci 57(2):166-175

\section{Publisher's Note}

Springer Nature remains neutral with regard to jurisdictional claims in published maps and institutional affiliations. 